\title{
Contrasting crustal sources for peraluminous granites of the segmented Montes de Toledo Batholith (Iberian Variscan Belt)
}

\author{
Carlos VILLASECA ${ }^{1 *}$, Cecilia PÉREZ-SOBA ${ }^{1}$, Enrique MERINO ${ }^{1}$, David OREJANA ${ }^{1}$, \\ José A. LÓPEZ-GARCÍA², Kjell BILLSTROM ${ }^{3}$
}

\author{
Department of P'etrology and Geochemistry, Centro mixto UCM-CSIC, Fandty of Geologital Sciences, Complutense University, 28040 \\ Madrid, Spain: granito@geo.ucm.es \\ 2 Department of Crystallography and.hineralogy, Fachlty of Geological Sciences, Complutense Lniversity, 28070.4fadrid. Spain \\ ${ }^{3}$ Swedish Museum of Vanural History: Box 50007, 10405 Stockholm, Sweden \\ * Corresponding muthor
}

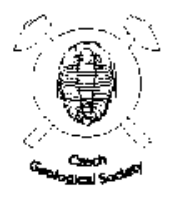

\begin{abstract}
The Variscan Montes de Toledo Batholith (MTB) is an E-W linear array of peraluminous granite plutons which is chemically segmented. The study is focused on the western segment of the MTB (W-MTB), mainly composed of granites with slightly lower $\mathrm{CaO}$ and higher $\mathrm{P}_{2} \mathrm{O}_{5}$ contents than associated eastern plutonic units and rearby S-type grarites, giving them a more pronounced peraluminous nature. The chemical contrast is also observed in isotopic composition, especially in radiogenic $\mathrm{Nd}$ and $\mathrm{Pb}$ ratios. The W-MTB grarites have higher initial $\varepsilon_{\mathrm{yd}} ; 5.0$ to -5.9$)$ and lower ${ }^{200} \mathrm{~Pb} / 204 \mathrm{~Pb}$ and ${ }^{208} \mathrm{~Pb} /{ }^{204} \mathrm{~Pb}$ ratios thar peralumijous types from the E-MTB segment. A mixed pelitic-greywackeous derivation from regional Neoproterozoic formations is suggested, whereas lower crustal and meta-igneous sources were involved in the origin of the easternmost MTB grarites. The presence of igneous muscovite together with coexisting andalusite and sillimanite in some of the strudied gramites suggests that solidus was reached at $650-700^{\circ} \mathrm{C}$ and depth comesponding to the pressure of $2-3 \mathrm{kbar}$.
\end{abstract}

Keywords: perahumirous granites, andhhusite, sillimanite, $\mathrm{Sr}-\mathrm{Nd}$-Pb isotopes, Iberian Variscan Belt

\section{Introduction}

The petrogenesis of strongly peraluminous granitoids has attracted attention of petrologists since early demonstration that granitic melts from which aluminium silicates can crystallize have been experimentally produced (e.g. Green 1976; Clemens and Wall 1981). Additionally, aluminium silicates have been found as unambiguous magmatic phenocrysts in volcanic rocks (e.g. Pichavant et al. 1988). The main problems in deciphering origin of peraluminous granites include: i) nature of the protolith, ii) incorporation of restites and presence of inherited components, iii) contamination during magma transport and emplacement, iv) superimposed magma differentiation processes (crystal fractionation, magma mixing, volatile exsolution, etc.), and v) pressure and temperature conditions during magma generation and crystallization.

The Variscan Iberian Belt is a sector of the European Variscides which is mainly composed of large granitic batholiths, particularly in its innermost area, that is, in the Central Iberian Zone (Fig. 1). Granitoids in this area constitute more than one third of the outcrops, and form one of the largest batholithic masses in the World. These granite plutons are predominantly peraluminous: metaaluminous varieties and related basic rocks are extremely scarce (e.g. Capdevila et al. 1973; Villaseca et al. 1998a; Bea et al. 1999). Most of these Variscan batholiths are complex assemblages where peraluminous and the more scarce metaluminous granite varieties appear together (c.g. Villaseca and Herreros 21)(W1). The studied Montes de Toledo Batholith (MTB) is a large batholithic array (around $200 \mathrm{~km}$ long) exclusively composed of peraluminous plutonic units (Fig. 1). Nevertheless distinct compositional differences between the western and eastern plutonic units suggest contrasting granite sources that gave rise to a chemically segmented granite batholith.

The existence of such a huge volume of peraluminous granitoids in the Variscan Iberian Belt has triggered extensive discussion about its genesis. Petrogenetic theories vary from i) important mantle input to its present-day composition, either by mixing (e.g. Dias and Leterrier 1994; Moreno Ventas et al. 1995) or by assimilation (c.g. Castro et al. 1999), to ii) mainly crustal recycling either by melting at mid-crustal levels (Bea et al. 2003) or by lower crustal derivation (Villaseca et al. 1999). Furthermore, it is difficult to assign S-type, I-type or transitional affinity to plutons with different degree of peraluminosity (c.g. Villaseca et al. 1998a).

In this paper we focus on the strongly peraluminous western segment of the Montes de Toledo Batholith. 
which has a clear S-type affinity. Most of the studied granites contain peraluminous minerals (andalusite, sillimanite, cordierite, muscovite, Al-rich biotite, tourmaline, etc.). The abundance of restite enclaves poses the problem of restite fractionation vs. other petrogenetic scenarios. This work provides mineralogical, geochemical and, for the first time, $\mathrm{Sr}-\mathrm{Nd}-\mathrm{Pb}$ isotopic information to motivate discussion on granite sources in this compositionally segmented peraluminous batholith.

\section{Geological setting}

The Montes de Toledo Batholith (MTB) is a linear array of approximately twenty granite plutons that is $\sim 200 \mathrm{~km}$ long and $20 \mathrm{~km}$ wide (in a W-E direction) from Belvís de Monroy (Caceres) to Madridejos (Toledo), and occupies an area of about $2000 \mathrm{~km}^{2}$ (Fig. 1). The best outcrop areas of this elongate granite batholith are their western and eastern segments, respectively, because its central part only crops out in deeply eroded river valleys. There the MTB is covered by Triassic sediments of the Tajo Basin and by coarse-grained alluvial fans from the Montes de Toledo Heights.

This plutonic array intruded into low-grade Neoproterozoic and Lower Palaeozoic metasedimentary rocks and generated remarkable contact metamorphic aureoles (IGME 1985, 1987, 1989). These units consist of alternating sandstone and shale, more than $4000 \mathrm{~m}$ thick, with interbedded conglomerate, calcareous mudstone and limestone of the so called "Schist-Crreywacke Formation", which is of Cambrian age in its upper part (Valdelacasa or Pusa Group - Valladares et al. 2002; Liñán et al. 2002). It is important to note the high phosphorous content of some metasediments in either Neoproterozoic or Lower Cambrian series (IGME 1987, 1989). The MTB crosscuts a set of open antiforms and synforms with NW-SE trending axes, related to the Variscan $\mathrm{D}_{1}$ compressional event (Ábalos et al. 2002). $D_{2}$ and $D_{3}$ structures are rare in the area although a conjugate system of NW-SE to NNW-SSE trending strike-slip dextral shear zones $\left(\mathrm{D}_{3}\right.$ phase) has been described ( $A$ balos et al. 2002).

The granitic MTB has been studied in detail in its eastern sector, that is, the Mora-Las Ventas Plutonic Complex, where an exhaustive petrological study (mineral chemistry, whole-rock geochemistry and Sr-Nd isotopes) has been performed (Andonaegui 1990; Villaseca et al. 1998a; Andonaegui and Villaseca 1998). The posttectonic peraluminous S-type granites from the eastern
MTB sector have been interpreted as derived from felsic meta-igneous sources (mainly from lower crustal levels), because outcropping metamorphic rocks and metasedimentary protoliths from low to middle and upper crust have a clearly different isotopic composition (Villaseca et al. 1998a; 1999). A preliminary Rb-Sr geochronological study gave an intrusion age of $320 \pm 8 \mathrm{Ma}$ (Andonaegui 1990). This age has to be considered with caution as the Variscan $\mathrm{D}_{3}$ phase has been dated to 313 to $325 \mathrm{Ma}$ elsewhere in the Central Iberian Zone (CIZ) (Dias et al. 1998; Fernández-Suárez et al. 200(0).

The western MTB segment studied in the present work comprises seven granitic plutons (Fig. 1) which were only incompletely described in the literature (IGME 1985 , 1987, 1989). Nevertheless, some preliminary petrological studies on the Aldeanueva de Barbarroya Pluton have been performed (Andonaegui and Barrera 1984), and the presence of two aluminium silicates (andalusite and sillimanite) in granites of this western segment of the MTB has been previously noted (Andonaegui and Barrera 1984; Fernández-Catuxo et al. 1995).

\section{Analytical techniques}

The major-element mineral compositions have been analysed at the Centro de Microscopía Electrónica "Luis Bru" (Complutense University of Madrid) using a Jeol JXA-8900 M electron microprobe with four wavelength dispersive spectromelers. Analytical conditions were an accelerating voltage of $15 \mathrm{kV}$, an electron beam current of $20 \mathrm{nA}$, and a beam diameter of $5 \mu \mathrm{m}$. Elements were counted for $10 \mathrm{~s}$ on the poak and $5 \mathrm{~s}$ on each background position. Corrections were made using the ZAF method. Representative major-element compositions of granite minerals are listed in Tables 1 to 3 .

The whole-rock major- and trace-element compositions were analysed at Actlabs ( $A$ ncaster, Ontario, Canada). The powdered samples were melted using $\mathrm{LiBO}_{2}$ and dissolved in TINO.. The solutions were analysed by inductively coupled plasma atomic emission spectrometry (ICP-AES) for major elements whereas trace elements were determined by ICP mass spectrometry (ICP-MS). Uncertainties in major elements are bracketed between 1 and $3 \%$, except for $\mathrm{MnO}(5-10 \%)$. The precision of ICP-MS analyses at low concentration levels has been evaluated from repeated analyses of the international standards BR, DR-N, UB-N, AN-G and GH. The precision for Rb, Si. 7r, Y, V, Hf and most of the REE is in

Fig. 1a - Sketch map of the Variscan Iberian Belt showing the location of the major granitic batholiths. Granite batholiths mentioned in the text are: $\mathrm{Al}=$ Albalá, $\mathrm{A}=$ Alburquerque, $\mathrm{CA}=$ Cabeza de Araya, $\mathrm{G}=$ Gata, $\mathrm{J}=\mathrm{Jálama}, \mathrm{MV}=$ Mora-Ventas, $\mathrm{TD}=$ Tormes Dome; granite cupolas are: $\mathrm{P}=$ Pedroso de Acim, $\mathrm{T}=$ Trujillo, $\mathrm{Tr}=$ El Trasquilón. $\mathbf{b}$ - Granite plutons of the western segment of the Montes de Toledo Batholith (W-MTB) with sample locations. 
(a) Galicia-Tras $3 / 7$

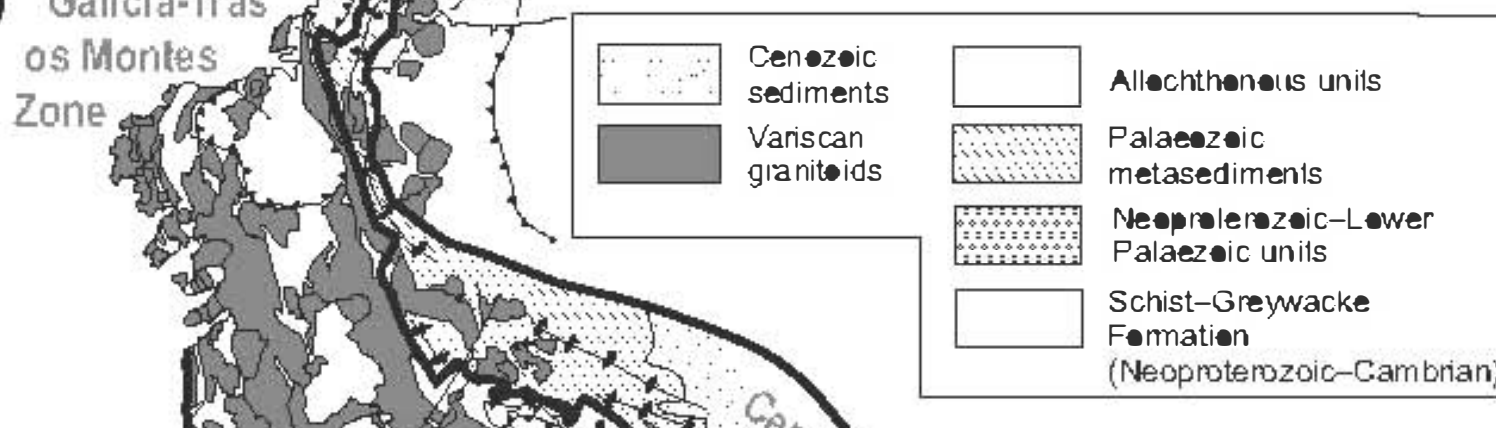

Fonnation

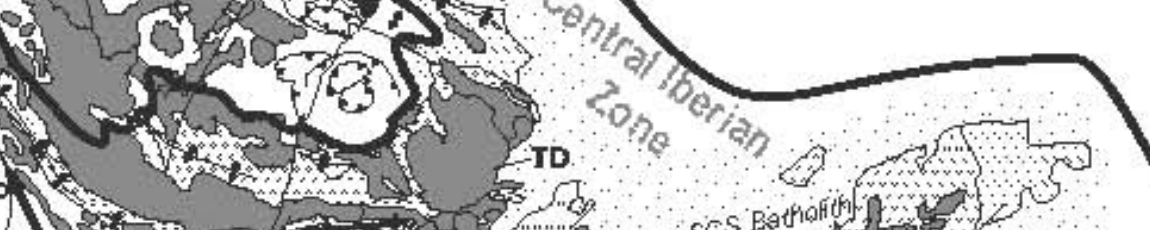

Portd
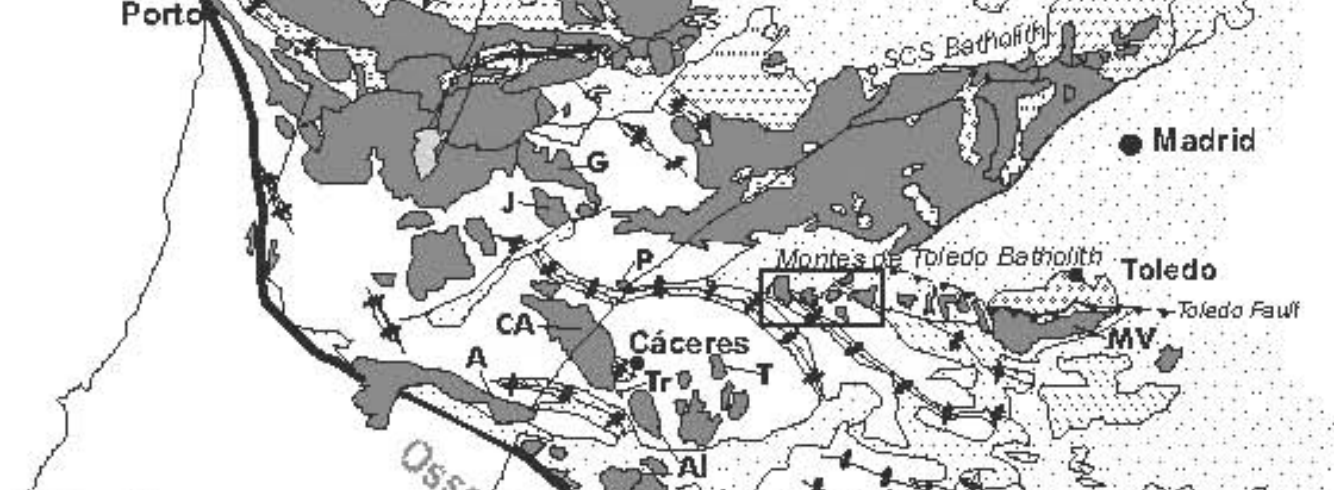

Lisboar
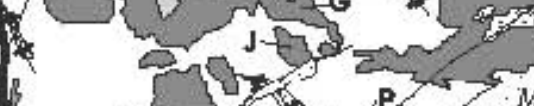

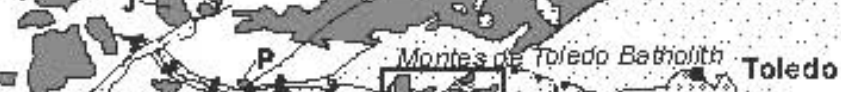
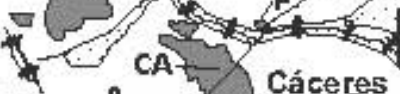

S. of
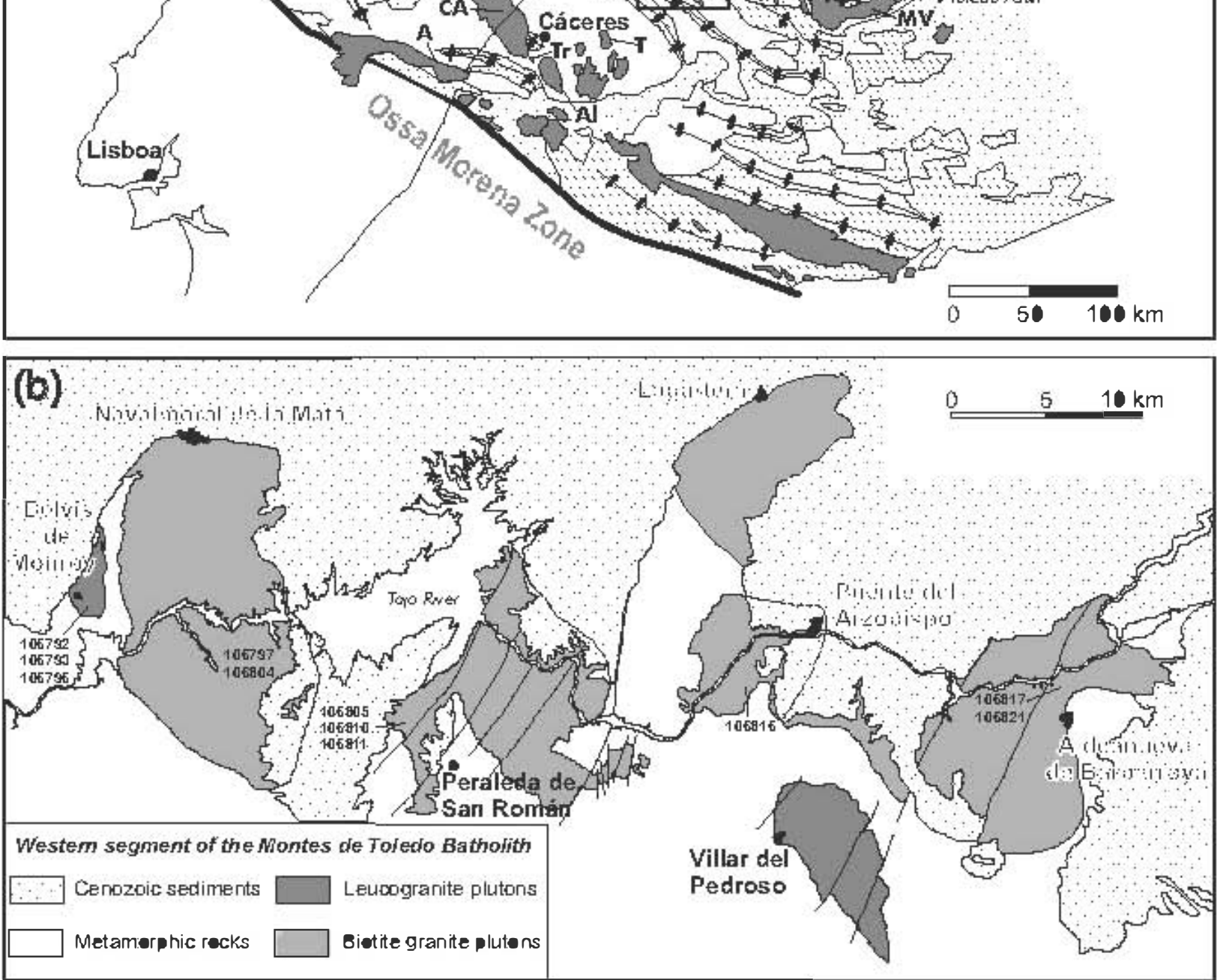
the range 1 to $5 \%$ whereas it varies from 5 to $10 \%$ for other trace elements including Tm. Some samples have concentrations of several elements below detection limits (V: $5, \mathrm{Cr}: 20, \mathrm{Sc}: 1 \mathrm{ppm}$ ) and all the granites have $\mathrm{Ni}<20$ $\mathrm{ppm}$. More information on the procedure, precision and accuracy of the Actlabs ICP-MS analyses can be found at $h t t p: / / w w w . a c t l a b s . c o m$.

The Sr-Nd isotopic analyses were carried out at the CAI de Geocronología y Geoquímica Isotópica, at the Complutense University of Madrid, using an automated VG Sector 54 multicollector thermal ionisation mass spectrometer. Analytical data were acquired in multidynamic mode. The analytical procedures used in this laboratory have been described elsewhere (Reyes et al. 1997). Repeated analyses of the NBS 987 standard gave ${ }^{87} \mathrm{Sr} /{ }^{86} \mathrm{Sr}=$ $0.710234 \pm 30(2 \sigma, \mathrm{n}=12)$ and for the $\mathrm{JM} \mathrm{Nd}$ standard the values of ${ }^{14} \mathrm{Nd} /{ }^{144} \mathrm{Nd}=0.511854 \pm 3(2 \sigma, \mathrm{n}=63)$ were obtained. The $2 \sigma$ error on $\varepsilon_{\mathrm{Id}}$ calculation is \pm 0.3 .

One whole-rock granite (from the eastern MTB) and $\mathrm{K}$-feldspar separates from three peraluminous granites (one from the western MTB and two from the Spanish Central System) were selected for $\mathrm{Pb}$ isotopic analyses at the Swedish Museum of Natural History (Stockholm) using a Finnigan MAT 261 TIMS with multicollector detection. The samples were dissolved with $\mathrm{HF}$ and $\mathrm{HNO}_{3}$ and a ${ }^{205} \mathrm{~Pb}$ spike was added to each of them. Lead was separated using cation exchange columns. The NBS 981 and 982 standards were used to evaluate instrumental fractionation and precision, the latter being about $0.1 \%$ for the isotopic ratios shown. Repeated analyses of international standard BCR-1 were used to monitor the accuracy.

\section{Petrography}

This study is mainly focused on five granite plutons cropping out from $\mathrm{W}$ to $\mathrm{E}$ in the following order: Belvís de Monroy, Navalmoral de la Mata, Peraleda de San Román, Puente del Arzobispo and Aldeanueva de Barbarroya (Fig. 1). Sample locations are shown in Fig. 1. Three samples of two-mica leucogranite were taken from the Belvís de Monroy Pluton, and they represent the most fractionated granite in this study. Samples from the Navalmoral de la Mata Pluton are biotite granites with variable amounts of $\mathrm{K}$-feldspar megacrysts, whereas biotite granites from the Puente del Arzobispo Pluton are more equigranular and include leucocratic varieties. The granites sampled from the Peraleda Pluton are slightly more mafic and have Al-rich minerals such as pinnitized cordierite and two aluminium silicates (andalusite and sillimanite). The fine-grained granodioritic facies of the Aldeanueva Pluton (also with Al-rich mineral phases) was only sampled for mineral chemistry, but the wholerock major-element chemical data from Andonaegui and Barrera (1984) have been considered in the geochemistry section for comparison.

All studied granites are peraluminous in composition and most of them have quartz, plagioclase, $\mathrm{K}$-feldspar and biotite as major rock-forming minerals. Biotite is the main mafic mineral in the studied granites; its amount varies from approx. $20 \mathrm{vol}$ \% (Aldeanueva granodioritic facies) to $1-5$ vol. \% (Belvís leucogranite). In the last mentioned two-mica leucogranite pluton, muscovite dominates over biotite.

Accessory amounts of cordierite, aluminium silicates, muscovite, tourmaline, apatite, zircon, monazite, ilmenite and xenotime appear in the MTB granites. It is interesting to note the presence of two polymorphs of the $\mathrm{Al}_{2} \mathrm{SiO}_{5}$ in two granite plutons, Peraleda and Aldeanueva, in which a clear textural evolution from andalusite to sillimanite is observed, rarely described in the literature.

The studied granites host different amounts of metasedimentary and restitic xenoliths, and locally some igneous felsic microgranular enclaves of cognate appearance, but they never contain mafic microgranular enclaves. The crustal xenoliths could be described as: i) metapsammitic xenoliths, ii) refractory metapelitic xenoliths, iii) surmicaceous enclaves, iv) globular quartz, and v) K-feldspar-rich lumps. Such a suite of metasedimentary lithologies is typical of S-type granites (Chappell et al. 1987; Barbero and Villaseca 1992). These inclusions are rarely larger than $25 \mathrm{~cm}$ in size, usually around $2-10 \mathrm{~cm}$, and could locally define enclave-rich sectors or contaminated facies.

\section{Mineral chemistry}

Plagioclase composition in studied granites ranges between $\mathrm{An}_{35}$ and $\mathrm{An}_{05}$, reaching albite in the leucogranites from the Belvís de Monroy Pluton (Fig. 2a). Zoning is poorly developed, but the most An-rich analyses are usually found in core zones. The $\mathrm{P}_{2} \mathrm{O}_{5}$ contents increase with the $\mathrm{Na}$ contents of the plagioclase, those from Belvís leucogranites show the highest concentrations (up to 0.91 wt. \%) (Fig. 2b). Plagioclase from granites of this MTB western sector is richer in $\mathrm{P}$ than that from other peraluminous S-type granites of central Spain (Fig. 2b).

$\mathrm{K}$-feldspar is weakly perthitic. Its composition varies between $\mathrm{Or}_{100}$ and $\mathrm{Or}_{78}$, being more Ab-rich in the Peraleda Pluton (Tab. 1). The $\mathrm{P}_{2} \mathrm{O}_{5}$ content is also high in the Belvís leucogranites (up to $0.92 \mathrm{wt}$. \%), exceeding concentrations in the perphosphorous Pedrobernardo granite from the Spanish Central System (SCS) (Bea et al. 1994) (Fig. 2c). 


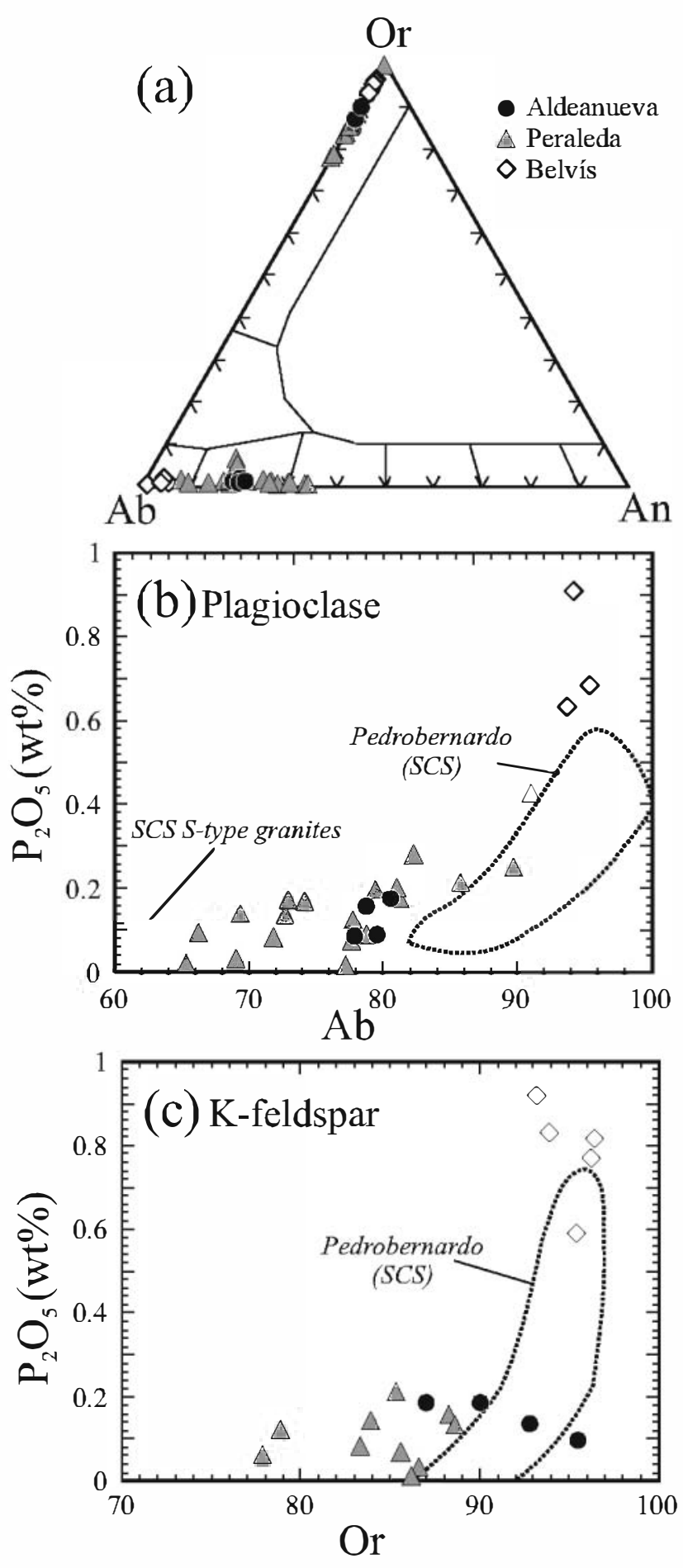

Biotite appears as subhedral crystals rich in mineral inclusions, usually accessory phases (Fig. 3a). It has Al-rich composition, and plots in peraluminous fields in biotite classification diagrams (e.g. Nachit et al. 1985; Abdel-Rahman 1994) similarly to other biotites from the SCS S-type granites (Fig. 4a). Biotite from the most felsic pluton of the MTB (the Belvís leucogranite) shows the highest $\mathrm{Al}$ and $\mathrm{F}$ contents (and, concomitantly, the lowest $\mathrm{Mg}$ and Ti contents) (Tab. 2).

Muscovite appears in most of the granites as late-magmatic anhedral crystals, sometimes rimming aluminium silicate grains, and usually in accessory amounts only. Nevertheless, in the Belvís leucogranites muscovite is the main mica, with larger size than associated biotite (Fig. 3b). In this two-mica leucogranite, muscovite crystals are remarkably euhedral (Fig. 3b). In granites where biotite dominates over muscovite, the larger muscovite crystals are those forming monocrystalline rims around andalusite, and are interpreted as magmatic according to the textural criteria reviewed by Clarke et al. (2005). There are no significant chemical differences between different textural types of muscovite and they all plot within igneous muscovite fields (Fig. 4b), including the fine-grained and flaky crystals of late-to-postmagmatic appearance. Similarly to biotite, muscovite of the Belvís leucogranites shows the highest $\mathrm{F}$ contents in the MTB (up to 0.86 wt. \%) (Tab. 2).

Cordierite is usually strongly transformed to secondary pinnite aggregates. Only in the fine-grained granodiorites of the Aldeanueva Pluton it appears as unaltered euhedral crystals of medium to coarse grain size (up to 2 $\mathrm{cm}$ ), hosting inclusions of quartz, biotite and scarce sillimanite. This suggests a non-cotectic growth but rather reaction with restitic or xenocrystic minerals immersed in the granite magma (similar to the CG1 type of Erdmann et al. 2004). Cordierite from the Aldeanueva Pluton has an $\mathrm{X}_{\mathrm{Mg}}$ of c. 0.57 with relatively low $\mathrm{Mn}$ and $\mathrm{Na}$ contents, although high enough to plot within the igneous field defined by the SCS cordierite-bearing granites (Fig. 5).

Andalusite appears as inclusions-free euhedral crystals of medium size in the Peraleda and Aldeanueva granites (Fig. 3c-d). It is of late-magmatic origin, occasionally closely associated with Ab-rich margins of plagioclase crystals. It seems to have crystallized close to magma solidus conditions as is the case of many andalusitebearing granites (Clarke et al. 2005). Andalusite has a thin rim of muscovite, but it maintains its original shape. Occasionally it shows a thin rim of fibrolitic sillimanite (always less than $100 \mu \mathrm{m}$ ) and an outer muscovite rim (Fig. 3c-d). Andalusite may show chemical zoning characterized by outward decreasing $\mathrm{FeO}$ content, in the range of 0.58 to $0.23 \mathrm{wt}$. \% (Fig. 6). This chemical zoning is less pronounced than in andalusites from other felsic

Fig. 2 Composition of feldspars from the W-MTB granites. a - Ternary Ab-Or-An diagram. $\mathbf{b}$ - Plagioclase composition: $\mathrm{P}_{2} \mathrm{O}_{5}$ vs Ab. c - K-feldspar composition: $\mathrm{P}_{2} \mathrm{O}_{5} v s$ Or. Fields for feldspars from the perphosphorous SCS Pedrobernardo Pluton are from Bea et al. (1994), whereas plagioclase from SCS S-type granites are from Villaseca et al. (unpublished data). 
Tab. 1 Selected representative EMP analyses of feldspars from the W-MTB granites

\begin{tabular}{|c|c|c|c|c|c|c|c|c|c|c|c|c|c|c|c|}
\hline \multirow{4}{*}{$\begin{array}{l}\text { Sample } \\
\text { Analy sis } \\
\text { Pluton } \\
\text { Mineral }\end{array}$} & 6795 & 106795 & 106795 & 106795 & 106795 & 106809 & 106809 & 106810 & 106809 & 106814 & 106814 & 106821 & 106821 & 106817 & 106821 \\
\hline & 45 & 55 & 77 & 79 & 82 & 57 & 61 & 92 & 60 & 35 & 24 & 38 & 43 & 70 & 86 \\
\hline & \multicolumn{5}{|c|}{ Belvís } & \multicolumn{6}{|c|}{ Peraleda } & \multicolumn{4}{|c|}{ Aldeanueva } \\
\hline & Pl & Pl & $\mathrm{Pl}$ & $\mathrm{Kfs}$ & $\mathrm{Kfs}$ & Pl & $\mathrm{Pl}$ & $\mathrm{Pl}$ & $\mathrm{Kfs}$ & $\mathrm{Kfs}$ & $\mathrm{Kfs}$ & $\mathrm{Pl}$ & $\mathrm{Pl}$ & $\mathrm{Kfs}$ & $\mathrm{Kfs}$ \\
\hline $\mathrm{SiO}_{2}$ & 65.66 & 64.98 & 65.57 & 62.53 & 62.34 & 66.47 & 62.31 & 58.75 & 64.50 & 64.63 & 65.91 & 62.64 & 62.84 & 63.46 & 63.93 \\
\hline $\mathrm{TiO}_{2}$ & $0 ?$ & 25 & n & 1 & 0.03 & 0.00 & .00 & 0 & O & 0.00 & 0. & 0 & 0.00 & 0 & 0.03 \\
\hline $\mathrm{Al}_{2} \mathrm{O}_{3}$ & 2060 & 20.94 & 21.17 & 18.92 & 19.60 & 20.87 & 24.31 & 26.17 & 18.78 & 18.92 & 19.34 & 23.46 & 23.33 & 19.07 & 19.15 \\
\hline $\mathrm{FeO}$ & 03 & 0.00 & 0.00 & 0.00 & 0.03 & 0.03 & 0.05 & 0.00 & 0.01 & 0.00 & 0.09 & 0.00 & 0.04 & 0.00 & 0.01 \\
\hline MnO & 0.00 & 0.04 & 0.00 & . & 0.01 & 0.04 & 0.08 & 0.00 & 0.04 & 0.03 & 0.00 & 0.00 & 0.00 & 0.00 & 0.02 \\
\hline $\mathrm{MgO}$ & 0.00 & 0.00 & 0.04 & 0.00 & 0.00 & 0.02 & 0.01 & 0.00 & 0.00 & 0.01 & 0.00 & 0.01 & 0.00 & 0.00 & 0.01 \\
\hline $\mathrm{CaO}$ & no & 0.71 & 0.74 & 0 & 0.00 & 1.55 & 5.55 & 6.96 & 0.00 & 0.04 & 0.00 & 4.08 & 4.20 & 0.02 & 0.06 \\
\hline $\mathrm{Na}_{2} \mathrm{O}$ & 10.59 & 10.05 & 11.00 & 0.42 & 0.76 & 10.70 & 8.53 & 7.73 & 1.27 & 1.83 & 2.53 & 9.19 & 8.75 & 0.79 & 1.41 \\
\hline $\mathrm{K}_{2} \mathrm{O}$ & 0.16 & 0.35 & 0.20 & 16.13 & 15.85 & 0.29 & 0.21 & 0.15 & 14.67 & 14.02 & 13.58 & 0.33 & 0.24 & 15.88 & 14.67 \\
\hline $\mathrm{P}_{2} \mathrm{O}_{5}$ & 4 & 91 & 69 & 77 & 0.92 & 0.43 & 0.14 & 0.10 & 0.16 & 0.08 & 0.06 & 0.16 & 0.09 & 0.14 & 0.19 \\
\hline Total & 98.00 & 98.41 & 99.48 & 98.78 & 99.55 & 100.42 & 101.31 & 99.95 & 99.53 & 99.75 & 101.59 & 99.96 & 99.52 & 99.43 & 99.51 \\
\hline$A b$ & 93.80 & 94.20 & 95.40 & 3.80 & 6.80 & 91.10 & 72.70 & 66.20 & 11.70 & 16.50 & 22.10 & 78.80 & 77.90 & 7.00 & 12.70 \\
\hline An & 5.30 & 3.70 & 3.50 & 0.00 & 0.00 & 7.30 & 26.10 & 33.00 & 0.00 & 0.20 & 0.00 & 19.30 & 20.70 & 0.10 & 0.30 \\
\hline Or & 0.90 & 2.20 & 1.10 & 96.20 & 93.20 & 1.60 & 1.20 & 0.80 & 88.30 & 83.30 & 77.90 & 1.90 & 1.40 & 92.80 & 87.00 \\
\hline
\end{tabular}

Tab. 2 Selected representative EMP analyses of micas from the W-MTB granites

\begin{tabular}{|c|c|c|c|c|c|c|c|c|c|c|c|c|c|c|c|}
\hline \multirow{4}{*}{$\begin{array}{l}\text { Sample } \\
\text { Analy sis } \\
\text { Pluton } \\
\text { Mineral }\end{array}$} & 106795 & 106795 & 106795 & 106795 & 106795 & 106809 & 106809 & 106809 & 106809 & 106809 & 106809 & 106817 & 106817 & 106821 & 106821 \\
\hline & 52 & 75 & 47 & 49 & 50 & 68 & 12 & 4 & 11 & 3 & 5 & 66 & 74 & 34 & 89 \\
\hline & \multicolumn{5}{|c|}{ Belvís } & \multicolumn{6}{|c|}{ Peraleda } & \multicolumn{4}{|c|}{ Aldeanueva } \\
\hline & $\mathrm{Bt}$ & $\mathrm{Bt}$ & Ms & Ms & Ms & $\mathrm{Bt}$ & $\mathrm{Bt}$ & $\mathrm{Bt}$ & Ms & Ms & Ms & $\mathrm{Bt}$ & $\mathrm{Bt}$ & Ms & Ms \\
\hline $\mathrm{SiO}_{2}$ & 35.84 & 35.63 & 46.02 & 45.63 & 46.23 & 35.17 & 35.50 & 36.25 & 45.66 & 46.03 & 44.50 & 34.65 & 34.85 & 46.66 & 45.83 \\
\hline $\mathrm{TiO}_{2}$ & 2.01 & 1.89 & 0.50 & 0.43 & 0.35 & 2.98 & 2.56 & 2.52 & 1.11 & 0.29 & 0.56 & 2.63 & 2.93 & 0.09 & 0.17 \\
\hline $\mathrm{Al}_{2} \mathrm{O}_{3}$ & 22.53 & 22.28 & 35.88 & 35.65 & 36.00 & 19.05 & 19.47 & 19.20 & 35.30 & 35.52 & 38.32 & 19.80 & 19.61 & 37.07 & 36.73 \\
\hline $\mathrm{FeO}$ & 19.10 & 20.30 & 1.51 & 1.41 & 1.38 & 20.66 & 18.05 & 18.19 & 0.73 & 1.03 & 0.54 & 19.96 & 18.43 & 1.13 & 0.79 \\
\hline MnO & 0.23 & 0.14 & 0.00 & 0.02 & 0.00 & 0.25 & 0.13 & 0.16 & 0.03 & 0.01 & 0.00 & 0.24 & 0.24 & 0.00 & 0.01 \\
\hline $\mathrm{MgO}$ & 4.10 & 3.02 & 0.65 & 0.77 & 0.64 & 7.54 & 8.21 & 8.62 & 0.65 & 0.69 & 0.51 & 7.44 & 7.83 & 0.83 & 0.67 \\
\hline $\mathrm{CaO}$ & 0.01 & 0.00 & 0.01 & 0.00 & 0.01 & 0.02 & 0.00 & 0.00 & 0.00 & 0.07 & 0.03 & 0.00 & 0.00 & 0.01 & 0.01 \\
\hline $\mathrm{Na}_{2} \mathrm{O}$ & 0.07 & 0.08 & 0.58 & 0.72 & 0.74 & 0.13 & 0.19 & 0.13 & 0.57 & 0.70 & 0.52 & 0.11 & 0.19 & 0.59 & 0.55 \\
\hline $\mathrm{K}_{2} \mathrm{O}$ & 9.55 & 9.42 & 9.59 & 9.80 & 9.45 & 9.50 & 9.59 & 9.57 & 10.62 & 10.76 & 9.91 & 9.61 & 9.55 & 9.90 & 10.06 \\
\hline $\mathbf{F}$ & 1.17 & 0.81 & 0.73 & 0.72 & 0.76 & 0.00 & 0.25 & 0.28 & 0.16 & 0.19 & 0.12 & 0.40 & 0.48 & 0.11 & 0.12 \\
\hline Total & 94.66 & 93.65 & 95.56 & 95.21 & 95.67 & 95.43 & 94.08 & 95.08 & 94.92 & 95.30 & 95.05 & 94.95 & 94.19 & 96.40 & 94.98 \\
\hline $\mathrm{Mg} /(\mathrm{Fe}+\mathrm{Mg})$ & 0.28 & 0.21 & 0.48 & 0.53 & 0.49 & 0.39 & 0.45 & 0.46 & 0.65 & 0.58 & 0.66 & 0.40 & 0.43 & 0.61 & 0.64 \\
\hline
\end{tabular}

granites of the Central Iberian Zone (CIZ) or from the granite pegmatites (Fernández-Catuxo et al. 1995; Clarke et al. 2005) (Fig. 6).

Sillimanite only appears in andalusite-bearing granites, and mostly forms thin rims surrounding andalusite.
Nevertheless, a second textural sillimanite type in these granites is the one associated with mica-rich enclaves or aggregates or even included in cordierite crystals as described previously (restites?). Sillimanite formed by andalusite transformation is invariably fibrolitic. Chemi- 


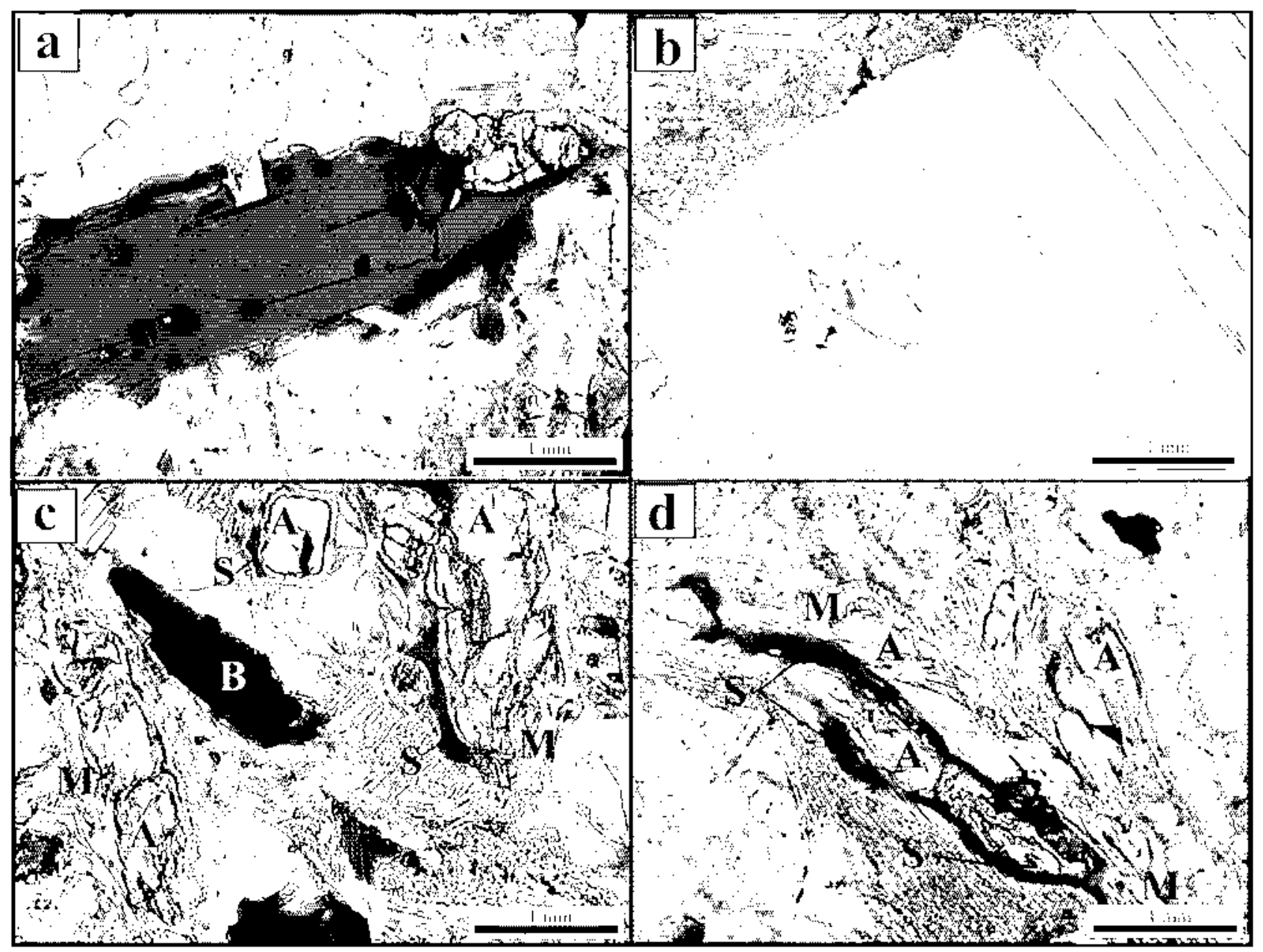

Fig. 3 Photomicrographs of granites from the W-MTB a - Inclusion-rich subhedral biotite crystal (106810 Peraleda granite) b - Large euhedral muscovite crystal enclosing brotite (106795 Belvis leucogranite) $\mathbf{c}-\mathbf{d}$-Andalusite crystals (A.) rimmed by fibrolitic sillimanite (S) and muscovite (M) (106809 and 106810 Peraleda granites, respectively)
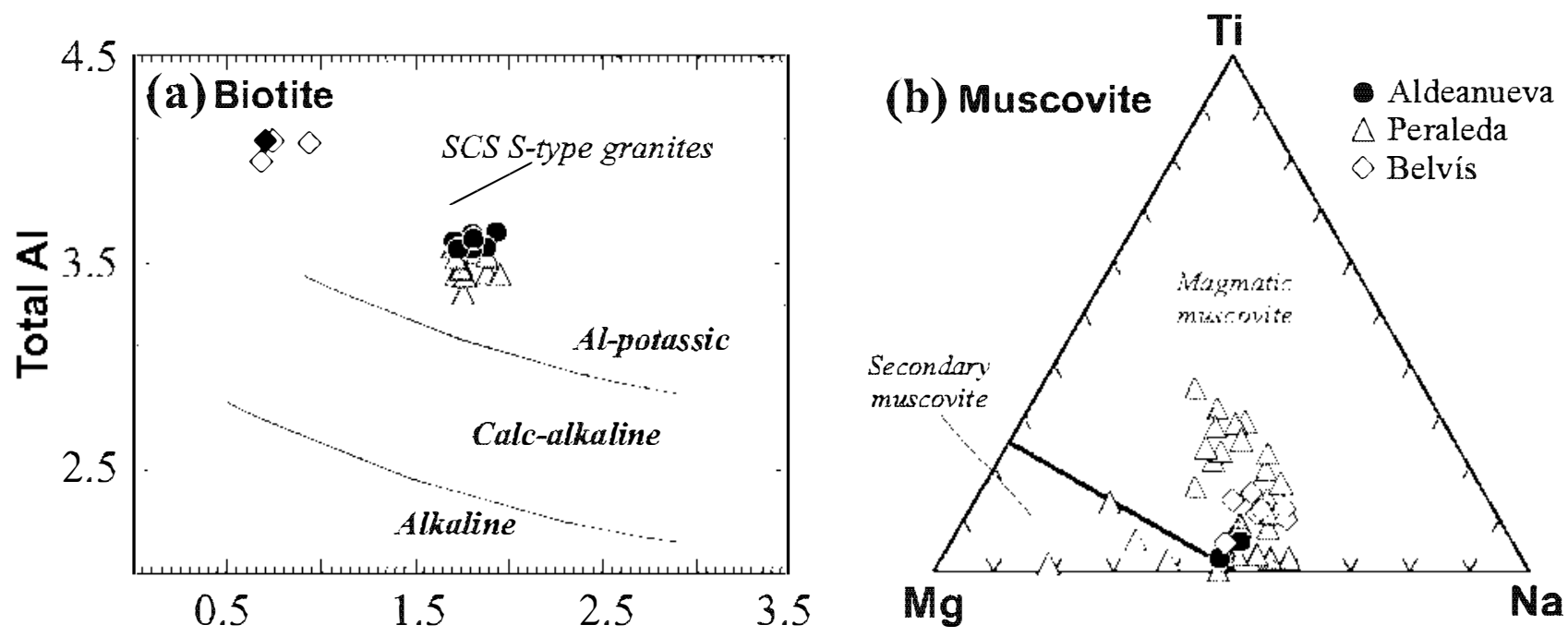

Fig. 4 Composition of micas from the W-MTB granites a - $\mathrm{Al}_{\mathrm{T}} \nu_{S} \mathrm{Mg}$ (atorns per $22 \mathrm{O}$ ) diagram for biotite Biotite compositional fields from different magmatic series are taken from Nachit et al (1985) Biotite compositional field of peraluminous S-type granites from the Sierra de Guadarrama (SCS granites) is taken from Villaseca and Barbero (1994) b - Mg-Ti-Na (atoms per 22 O) diagram for muscovite Magmatic and secondary muscovite fields are taken frorn Miller et al (1981) 


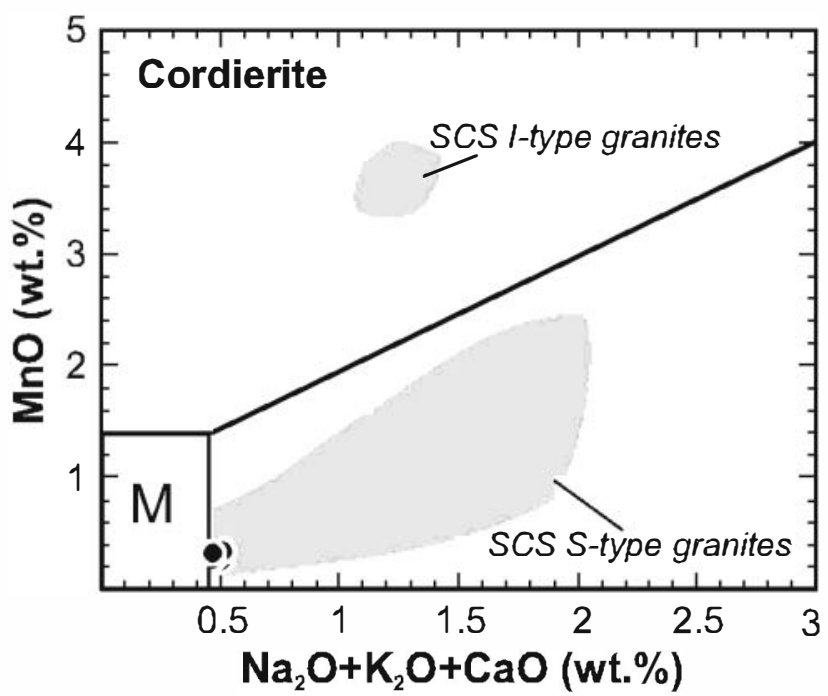

Fig. 5 Composition of cordierite in the discriminant diagram $\left(\mathrm{Na}_{2} \mathrm{O}+\right.$ $\mathrm{K}_{2} \mathrm{O}+\mathrm{CaO}$ ) vs $\mathrm{MnO}$. Compositional fields for metamorphic ( $\mathrm{M}$ field) and igneous (S- and I-type granites) cordierite come from data of Barbero and Villaseca (1992) and Villaseca and Barbero (1994). Within the M field are also included cordierites from anatectic leucogranites and restite-rich granites of migmatite-granulite terranes (Barbero and Villaseca 1992).

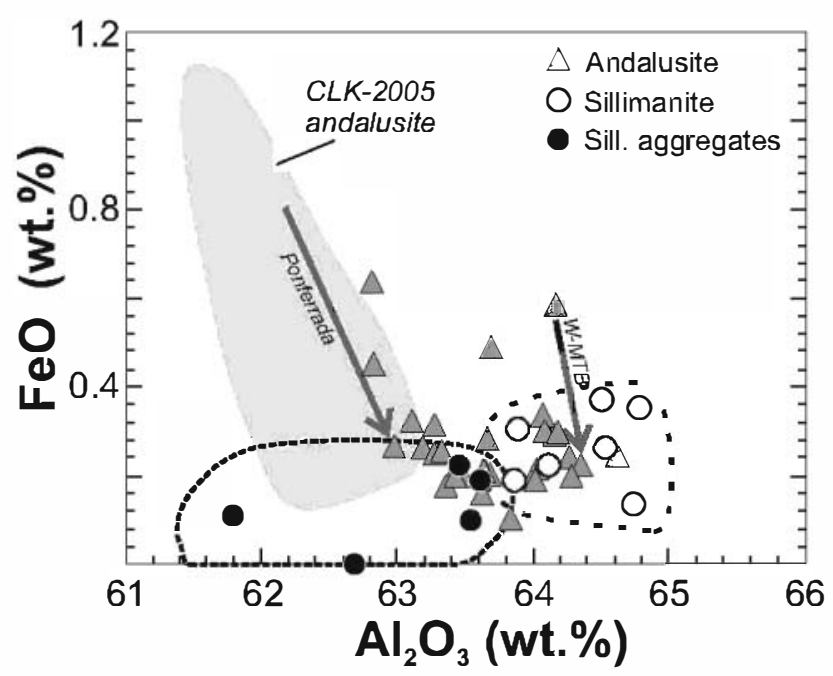

Fig. 6 Composition of aluminium silicates from the W-MTB granites in the $\mathrm{Al}_{2} \mathrm{O}_{3}$ vs $\mathrm{FeO}$ diagram. Arrows show core to rim zoning of an andalusite crystal from a W-MTB granite (Peraleda), together with andalusite zoning in another CIZ granite - the Ponferrada granite (Fernández-Catuxo et al. 1995). Andalusite compositional field from Clarke et al. (2005) (CLK-2005) is also included.

Tab. 3 Selected representative EMP analyses of minerals from the W-MTB granites

\begin{tabular}{|c|c|c|c|c|c|c|c|c|c|c|c|c|c|}
\hline \multirow{4}{*}{$\begin{array}{l}\text { Sample } \\
\text { Analysis } \\
\text { Pluton } \\
\text { Mineral }\end{array}$} & 106821 & 106821 & 106821 & 106814 & 106814 & 106814 & 106814 & 106809 & 106817 & 106817 & 106809 & 106810 & \multirow{4}{*}{$\begin{array}{c}106817 \\
59 \\
\text { Aldeanueva } \\
\text { Ap }\end{array}$} \\
\hline & 35 & 36 & 83 & 4 & 11 & 24 & 28 & 55 & 73 & 58 & 59 & 96 & \\
\hline & \multicolumn{3}{|c|}{ Aldeanueva } & \multicolumn{5}{|c|}{ Peraleda } & \multicolumn{2}{|c|}{ Aldeanueva } & \multicolumn{2}{|c|}{ Peraleda } & \\
\hline & Crd & Crd & Crd & And & And & And & Sil & Sil & And & Sil & Ap & Ap & \\
\hline $\mathrm{SiO}_{2}$ & 47.44 & 47.39 & 46.64 & 36.63 & 37.05 & 36.63 & 35.82 & 36.80 & 36.23 & 36.25 & 0.00 & 0.03 & 0.03 \\
\hline $\mathrm{TiO}_{2}$ & 0.00 & 0.00 & 0.00 & 0.00 & 0.11 & 0.15 & 0.05 & 0.00 & 0.07 & 0.01 & 0.00 & 0.00 & 0.00 \\
\hline $\mathrm{Al}_{2} \mathrm{O}_{3}$ & 33.13 & 33.31 & 33.48 & 62.82 & 62.81 & 64.16 & 64.46 & 62.67 & 64.17 & 63.85 & 0.00 & 0.00 & 0.00 \\
\hline $\mathrm{FeO}$ & 9.40 & 9.07 & 8.88 & 0.45 & 0.64 & 0.58 & 0.39 & 0.00 & 0.30 & 0.19 & 0.39 & 0.33 & 0.34 \\
\hline MnO & 0.25 & 0.34 & 0.27 & 0.00 & 0.00 & 0.00 & 0.04 & 0.00 & 0.01 & 0.00 & 0.61 & 0.56 & 0.47 \\
\hline $\mathrm{MgO}$ & 6.74 & 7.15 & 6.91 & 0.00 & 0.14 & 0.12 & 0.10 & 0.00 & 0.04 & 0.01 & 0.00 & 0.01 & 0.06 \\
\hline $\mathrm{CaO}$ & 0.00 & 0.01 & 0.01 & 0.01 & 0.00 & 0.00 & 0.05 & 0.00 & 0.00 & 0.01 & 53.33 & 53.73 & 56.35 \\
\hline $\mathrm{Na}_{2} \mathrm{O}$ & 0.5 & 0.49 & 0.48 & 0.00 & 0.04 & 0.00 & 0.03 & 0.02 & 0.02 & 0.04 & 0.20 & 0.23 & 0.12 \\
\hline $\mathrm{K}_{2} \mathrm{O}$ & 0.00 & 0.02 & 0.00 & 0.00 & 0.00 & 0.00 & 0.16 & 0.06 & 0.00 & 0.00 & 0.02 & 0.00 & 0.01 \\
\hline $\mathrm{P}_{2} \mathrm{O}_{5}$ & - & - & - & - & - & - & - & - & - & - & 41.64 & 41.39 & 42.00 \\
\hline F & - & - & - & - & - & - & - & - & - & - & 4.04 & 4.38 & 4.11 \\
\hline Total & 97.49 & 97.79 & 96.68 & 100.05 & 100.81 & 101.77 & 101.14 & 99.93 & 100.85 & 100.38 & 100.23 & 100.66 & 101.80 \\
\hline
\end{tabular}

cally it is slightly richer in $\mathrm{Al}$ than that from restitic environments (aggregates, enclaves, inclusions) (Fig. 6).

Apatite is very common (except in the Belvís leucogranites), forming euhedral crystals. Its $\mathrm{F}$ content (up to $4.8 \mathrm{wt} . \%)$ is noteworthy as it is higher than that from the SCS S-type granites (2.6 to $4.0 \mathrm{wt}$. \% F; Clarke et al. 2005) (Tab. 3).

\section{Geochemistry}

\subsection{Major and trace elements}

Major- and trace-element compositions of the studied granites are listed in Tab. 4. Major-element contents 
Tab. 4 Whole-rock major (wt. \%) and trace-element (ppm) compositions of the western MTB granites

\begin{tabular}{|c|c|c|c|c|c|c|c|c|c|c|}
\hline \multirow{2}{*}{$\begin{array}{l}\text { Sample } \\
\text { Pluton }\end{array}$} & 106811 & 106810 & 106805 & 106797 & 106804 & 106815 & 106816 & 106792 & 106796 & 106793 \\
\hline & \multicolumn{3}{|c|}{ Peraleda } & \multicolumn{2}{|c|}{ N. Mata } & \multicolumn{2}{|c|}{ P. Arzobispo } & \multicolumn{3}{|c|}{ Belvís } \\
\hline $\mathrm{SiO}_{2}$ & 68.67 & 69.96 & 70.21 & 71.52 & 71.33 & 72.45 & 74.05 & 73.34 & 74.08 & 74.51 \\
\hline $\mathrm{TiO}_{2}$ & 0.58 & 0.53 & 0.44 & 0.55 & 0.39 & 0.27 & 0.27 & 0.04 & 0.10 & 0.03 \\
\hline $\mathrm{Al}_{2} \mathrm{O}_{3}$ & 15.69 & 15.41 & 15.47 & 13.71 & 15.11 & 14.38 & 14.29 & 15.17 & 14.87 & 14.85 \\
\hline $\mathrm{Fe}_{2} \mathrm{O}_{3}$ & 3.57 & 3.01 & 2.58 & 3.40 & 2.36 & 1.68 & 1.88 & 0.50 & 0.90 & 0.37 \\
\hline MnO & 0.05 & 0.04 & 0.03 & 0.04 & 0.03 & 0.02 & 0.03 & 0.05 & 0.02 & 0.02 \\
\hline $\mathrm{MgO}$ & 1.25 & 1.04 & 0.93 & 0.84 & 0.67 & 0.50 & 0.56 & 0.09 & 0.19 & 0.06 \\
\hline $\mathrm{CaO}$ & 1.67 & 1.40 & 1.06 & 1.34 & 0.85 & 0.61 & 0.57 & 0.39 & 0.46 & 0.41 \\
\hline $\mathrm{Na}_{2} \mathrm{O}$ & 3.47 & 3.08 & 3.04 & 2.57 & 3.07 & 3.07 & 2.98 & 4.11 & 3.77 & 4.30 \\
\hline $\mathrm{K}_{2} \mathrm{O}$ & 3.84 & 4.56 & 4.79 & 4.63 & 5.43 & 4.75 & 4.61 & 3.84 & 3.96 & 3.68 \\
\hline $\mathrm{P}_{2} \mathrm{O}_{5}$ & 0.39 & 0.41 & 0.47 & 0.29 & 0.38 & 0.27 & 0.39 & 0.85 & 0.63 & 0.76 \\
\hline LOI & 0.83 & 0.84 & 1.27 & 0.71 & 0.78 & 1.05 & 1.02 & 0.99 & 1.19 & 0.94 \\
\hline Total & 100.00 & 100.30 & 100.30 & 99.62 & 100.40 & 99.29 & 100.70 & 99.37 & 100.20 & 99.92 \\
\hline A/CNK & 1.22 & 1.23 & 1.28 & 1.17 & 1.21 & 1.27 & 1.31 & 1.30 & 1.31 & 1.26 \\
\hline $\mathrm{Ba}$ & 496 & 549 & 441 & 493 & 474 & 254 & 243 & 16 & 63 & 16 \\
\hline $\mathbf{R b}$ & 270 & 277 & 255 & 260 & 263 & 414 & 393 & 569 & 459 & 430 \\
\hline $\mathrm{Sr}$ & 177 & 170 & 126 & 105 & 94 & 63 & 49 & 48 & 97 & 123 \\
\hline $\mathrm{Pb}$ & 24 & 25 & 30 & 29 & 33 & 26 & 27 & 10 & 18 & 10 \\
\hline Th & 17.8 & 18.4 & 12.1 & 17.9 & 13.2 & 14.2 & 15.2 & 0.73 & 1.9 & 0.59 \\
\hline $\mathbf{U}$ & 9.67 & 8.64 & 7.13 & 4.83 & 6.28 & 11.4 & 3.28 & 13.5 & 6.82 & 11.5 \\
\hline $\mathrm{Zr}$ & 198 & 165 & 150 & 175 & 128 & 104 & 104 & 28 & 44 & 20 \\
\hline $\mathrm{Nb}$ & 10.9 & 10.1 & 9.8 & 12.4 & 10.4 & 8.5 & 14.0 & 14.4 & 13.5 & 11.7 \\
\hline Y & 19.9 & 17.5 & 15.6 & 22.9 & 15.3 & 9.2 & 13.5 & 2.1 & 7.3 & 2.9 \\
\hline $\mathrm{Sc}$ & 7 & 5 & 5 & 7 & 5 & 3 & 4 & 1 & 2 & $<1$ \\
\hline $\mathbf{v}$ & 43 & 34 & 35 & 32 & 27 & 15 & 18 & $<5$ & $<5$ & $<5$ \\
\hline $\mathrm{Cr}$ & 140 & 80 & 30 & 30 & 40 & 30 & 60 & $<20$ & 30 & $<20$ \\
\hline $\mathrm{Ga}$ & 26 & 23 & 22 & 21 & 20 & 25 & 25 & 21 & 21 & 20 \\
\hline Ta & 1.10 & 1.23 & 1.42 & 1.67 & 1.67 & 1.83 & 2.52 & 4.98 & 5.34 & 5.29 \\
\hline $\mathrm{Hf}$ & 5.3 & 4.5 & 4.1 & 5.3 & 3.4 & 3 & 3.3 & 1.4 & 1.6 & 1.3 \\
\hline Cs & 19.4 & 18.6 & 20.6 & 14.5 & 19.3 & 45.9 & 29.2 & 13.7 & 70.6 & 16.9 \\
\hline Sn & 7 & 7 & 9 & 9 & 11 & 34 & 24 & 34 & 50 & 23 \\
\hline La & 43.20 & 37.80 & 26.40 & 35.60 & 26.20 & 19.20 & 21.10 & 1.73 & 4.91 & 1.46 \\
\hline $\mathrm{Ce}$ & 100.00 & 85.50 & 60.50 & 83.50 & 60.00 & 48.70 & 53.00 & 3.84 & 11.30 & 3.23 \\
\hline $\operatorname{Pr}$ & 11.10 & 9.63 & 6.86 & 9.43 & 6.81 & 5.73 & 6.03 & 0.42 & 1.30 & 0.35 \\
\hline Nd & 44.90 & 39.10 & 27.90 & 37.10 & 27.60 & 23.00 & 23.60 & 1.62 & 5.38 & 1.47 \\
\hline Sm & 7.68 & 7.15 & 5.62 & 7.30 & 5.18 & 5.13 & 5.41 & 0.37 & 1.34 & 0.38 \\
\hline Eu & 0.99 & 0.96 & 0.86 & 0.97 & 0.84 & 0.50 & 0.45 & 0.03 & 0.15 & 0.03 \\
\hline Gd & 5.64 & 5.47 & 4.97 & 5.95 & 4.26 & 3.66 & 4.20 & 0.38 & 1.45 & 0.41 \\
\hline $\mathrm{Tb}$ & 0.80 & 0.76 & 0.73 & 0.87 & 0.63 & 0.49 & 0.62 & 0.07 & 0.28 & 0.09 \\
\hline Dy & 4.01 & 3.47 & 3.29 & 4.42 & 3.02 & 2.19 & 2.90 & 0.38 & 1.47 & 0.51 \\
\hline Но & 0.64 & 0.57 & 0.49 & 0.74 & 0.50 & 0.31 & 0.44 & 0.06 & 0.20 & 0.08 \\
\hline $\mathrm{Er}$ & 1.70 & 1.48 & 1.19 & 2.01 & 1.33 & 0.77 & 1.10 & 0.15 & 0.48 & 0.21 \\
\hline $\mathrm{Tm}$ & 0.24 & 0.20 & 0.17 & 0.27 & 0.18 & 0.10 & 0.15 & 0.02 & 0.07 & 0.03 \\
\hline $\mathbf{Y b}$ & 1.52 & 1.23 & 1.01 & 1.69 & 1.12 & 0.64 & 0.94 & 0.13 & 0.39 & 0.18 \\
\hline Lu & 0.22 & 0.18 & 0.13 & 0.24 & 0.15 & 0.09 & 0.13 & 0.02 & 0.05 & 0.02 \\
\hline
\end{tabular}




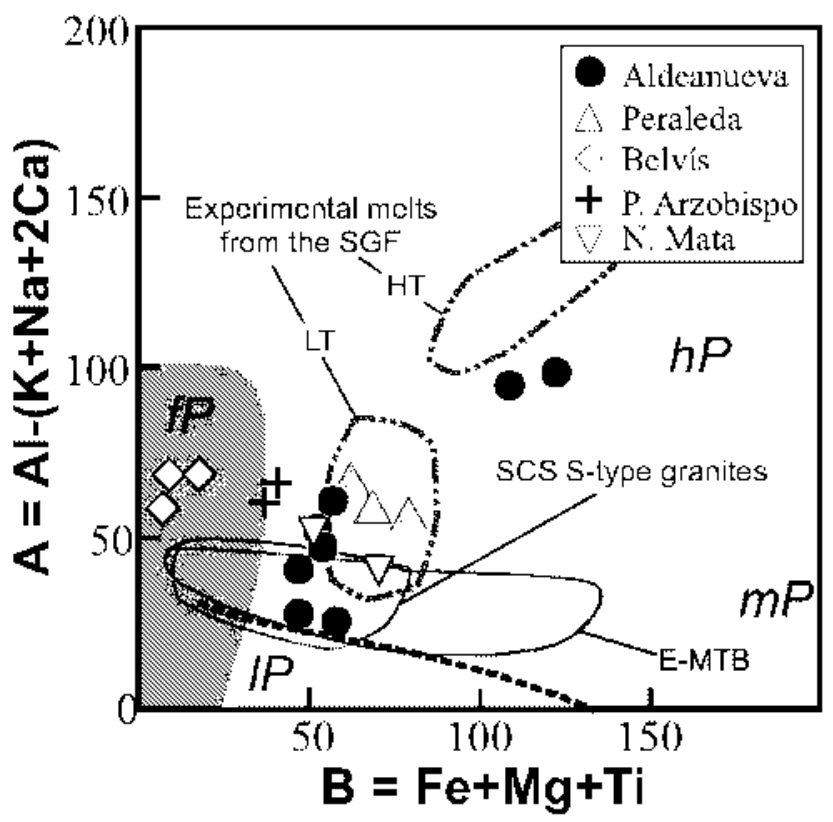

of granitoids from the Aldeanueva Pluton (takes from Andonaegui and Barrera 1984) have only been included in Fig. 7. The MTB granites range in $\mathrm{SiO}$, from 68.3 to $74.5 \mathrm{wt}$. \%. They all plot in subalkaline fields in the $\mathrm{SiO}_{2}$ versus $\mathrm{Na} \mathrm{O}+\mathrm{K}_{2} \mathrm{O}$ diagram and are peraluminous. In the A-B diagram (Debon and Le Fort 1983; modified by Villaseca et al. 1998 b; Fig. 7) samples from the most felsic pluton (Belvis de Monroy) fall in the $f P$ field. whereas other samples plot between the highly and

岁

Fig. 7 Composition of the W-MTB granites in the A-B diagram of Debon and Le Fort (1983), modified by Villaseca et al. (1998b). Compositional fields of the Mora-Ventas Batholith (E-MTB) and the SCS S-type granites are taken from Andonaegui (1990) and Villaseca et al. (1998b), respectively. In this diagram are also plotted chemical data from the Aldeanueva Pluton (Andonaegui and Barrera 1984). Fields of partial melting experiments at low $\left(1000-1100^{\circ} \mathrm{C}\right.$ ) and high temperature $\left(1200{ }^{\circ} \mathrm{C}\right)$ of schist-greywacke mixtures from the SGF are taken from Fernández et al. (2008).
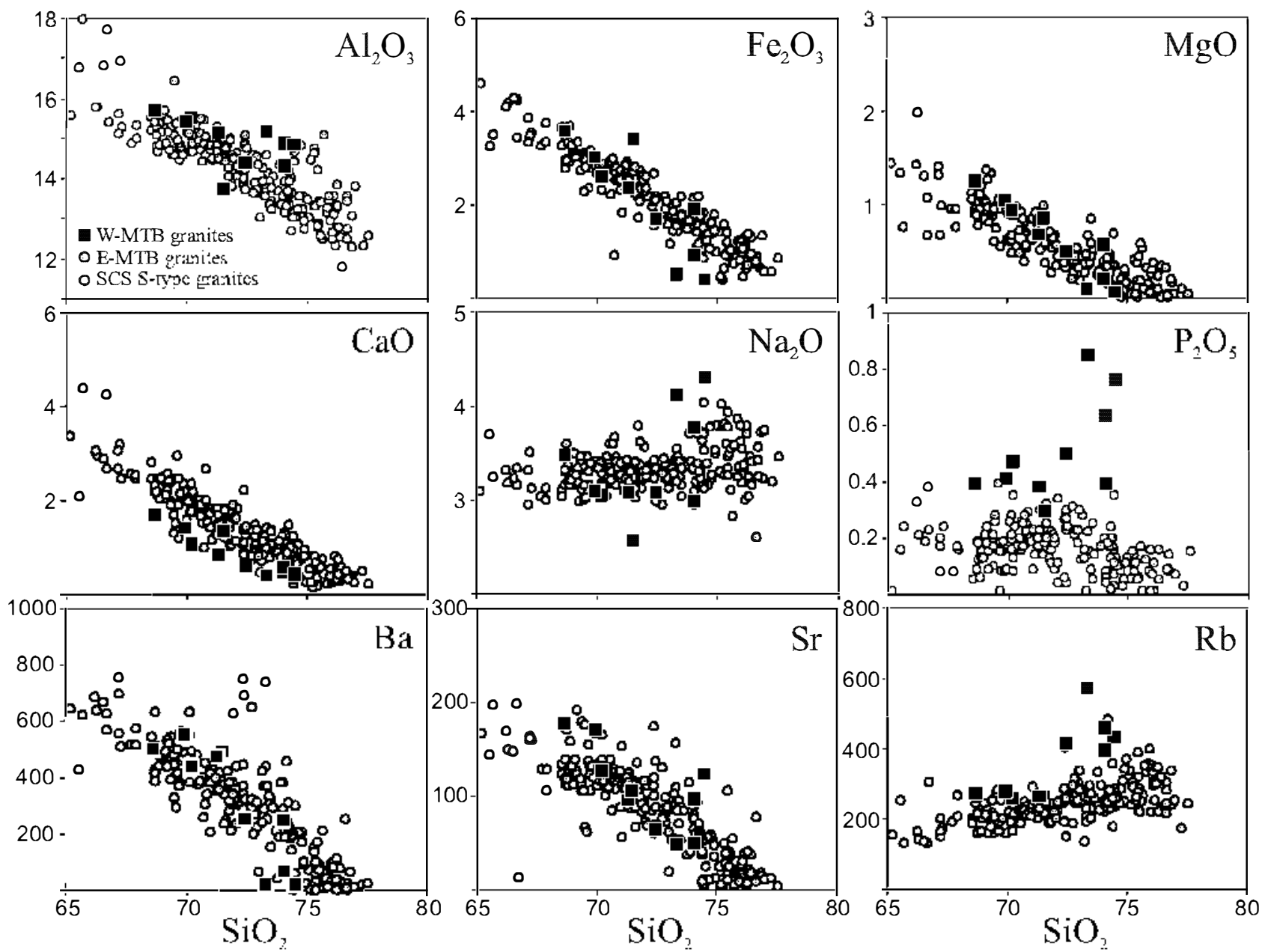

Fig. 8 Selected major (wt. \%) and trace (ppm) element variation diagrams for the W-MTB granites (Tab. 4). Data for S-type granites from the Mora-Ventas Batholith (E-MTB) and the SCS are taken from Andonaegui (1990) and Villaseca et al. (1998b), respectively. 
moderately peraluminous fields (Peraleda granites plot in the $h P$ field) with a higher peraluminosity than S-type granites either from the eastern MTB or from the SCS. In variation diagrams using $\mathrm{SiO}_{2}$ (Fig. 8), the studied MTB granites define a nearly continuous trend of decreasing $\mathrm{Fe}_{2} \mathrm{O}_{3}, \mathrm{MgO}, \mathrm{Al}_{2} \mathrm{O}_{3}, \mathrm{TiO}_{2}, \mathrm{CaO}$ values and increasing $\mathrm{P}_{2} \mathrm{O}_{5}$ contents. It is remarkable that the $\mathrm{CaO}$ and $\mathrm{P}_{2} \mathrm{O}_{5}$ contents are lower and higher, respectively, in the western MTB granites when compared with S-type granites from central Spain (eastern MTB and SCS) (Fig. 8). This is in agreement with the higher peraluminosity of the western MTB granites and this suggests different crustal sources for the segmented batholith. Moreover, the Belvis leucogranites show a marked perphosphorous trend, with $\mathrm{P}$ mainly hosted in feldspars, which is typical of some peraluminous granites of the south-western Central-Iberian Zone (Cabeza de Araya Batholith: Bea et al. 1992; Gata Plutonic Complex: Hassan Mohamud et al. 2002; Jálama and Alburquerque batholiths: Ramírez and Menéndez 1999) (for locations see Fig. 1).

There are no major differences in trace-element contents with respect to other S-type granite suites from central Spain (except for slightly higher Rb contents, Fig. 8). The studied MTB granites define a trend of decreasing $\mathrm{Ba}, \mathrm{Sr}, \mathrm{Zr}$, and, to a lesser degree, $\mathrm{Y}$ and HREE with silica concentration suggesting feldspar, biotite and accessory minerals fractionation. The lowest $\mathrm{Ba}, \mathrm{Sr}, \mathrm{Cr}, \mathrm{V}$, Sc, Zr, Hf, Y, HREE and Th contents are those observed in the Belvís leucogranites, which, by contrast, show the highest $\mathrm{P}$ and $\mathrm{Rb}$ values. The lack of increase in $\mathrm{Th}$, $\mathrm{Y}$ and HREE contents with $\mathrm{SiO}_{2}$ is a feature typical of S-type granite suites (e.g. Villaseca et al. 1998a).

Chondrite-normalized REE patterns for the western MTB granites are very similar to each other without significant degree of REE fractionation with increasing $\mathrm{SiO}_{2}$ contents (Fig. 9a), although the magnitude of the negative Eu anomaly (expressed as the $\mathrm{Eu} / \mathrm{Eu}^{*}$ ratios) increases from 0.33 (biotite granites of the Navalmoral and Peraleda plutons) to 0.15 (Belvís leucogranites). The relatively flat HREE patterns of all these MTB granites, with $(\mathrm{Gd} / \mathrm{Yb})_{1 \mathrm{~T}}$ values in the range of 4.6 to 1.8 , suggest an absence of garnet in the source and, therefore, middle crustal levels of partial melting. This feature is common to many strongly peraluminous granites (e.g. Rossi et al. 2002).

Multi-element patterns for the different MTB granites normalized by Bulk Continental Crust vary between flat crust-like compositions (biotite granites) and more spiky and depleted behaviour (leucogranites) (Fig. 9b). These patterns are very similar to the Lachlan Fold Belt cordierite-bearing S-type granites and to the Himalayan leucogranites, respectively (Kemp and Hawkesworth 2003). In addition, differences appear between individual leucogranite types (the Belvís leucogranites are much

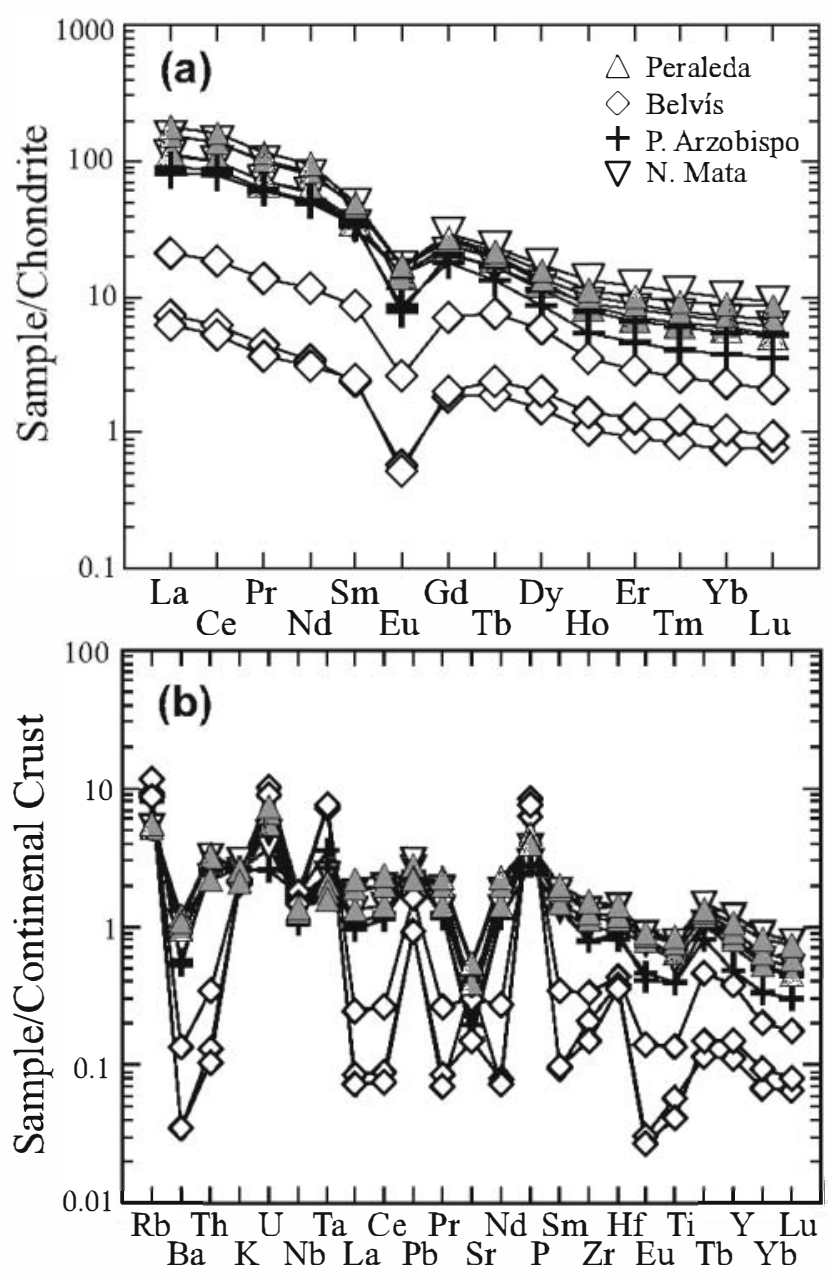

Fig. 9 Trace-element composition of the W-MTB granites. a - Chondrite-normalized REE patterns for the W-MTB granites. Normalizing values from Sun and McDonough (1989). b - Trace-element pattern s for the W-MTB granites normalized by the Bulk Continental Crust (Rudnick and Gao 2003).

more fractionated and markedly richer in phosphorous and depleted in lead than Himalayan types).

\section{$6.2 \mathrm{Sr}-\mathrm{Nd}-\mathrm{Pb}$ isotopes}

The $\mathrm{Sr}$ and $\mathrm{Nd}$ isotopic ratios were corrected to a representative Variscan age of $300 \mathrm{Ma}$. Analysed granites from the western MTB show a large variation in initial ${ }^{87} \mathrm{Sr} /{ }^{86} \mathrm{Sr}$ ratios $(0.7060$ to 0.7133$)$ whereas initial $\varepsilon_{\text {Id }}$ values are much less variable between -5.0 and -5.9 (Tab. 5; Fig. 10). The granites from the eastern MTB show slightly lower $\varepsilon_{\mathrm{Id}}$ values, from -5.7 to -6.6 , similar to values for the SCS S-type granites (Villaseca et al. 1998a) (Fig. 10). The only Sr-Nd isotopic data for other perphosphorous granites intruding the Schist-Greywacke Formation are those from the Alburquerque Batholith, 
Tab. $5 \mathrm{Sr}$ and Nd isotope data and concentrations (ppm) of the W-MTB granites

\begin{tabular}{|c|c|c|c|c|c|c|c|c|c|c|c|c|}
\hline Sample & Pluton & $\mathrm{Rb}$ & $\mathrm{Sr}$ & ${ }^{87} \mathrm{Rb} /{ }^{86} \mathrm{Sr}$ & ${ }^{87} \mathrm{Sr} /{ }^{86} \mathrm{Sr}$ & $\left({ }^{87} \mathrm{Sr} /{ }^{86} \mathrm{Sr}\right)_{3 \infty}$ & $\mathrm{Sm}$ & Nd & $\mathrm{Sm} / \mathrm{Nd}$ & ${ }^{147} \mathrm{Sm} /{ }^{144} \mathrm{Nd}$ & ${ }^{143} \mathrm{No}$ & $\varepsilon(\mathrm{Nd})_{300}$ \\
\hline 106792 & \multirow{3}{*}{ Belvís } & 569 & 48 & .808 & $0.856018 \pm 06$ & 0.707421 & 0.37 & 1.62 & 0.2284 & & & \\
\hline 106793 & & 430 & 123 & 10.171 & $0.760438 \pm 05$ & 0.717019 & 0.38 & 1.47 & 0.2585 & & & \\
\hline 106796 & & 459 & 97 & 13.788 & $0.776847 \pm 05$ & 0.717983 & 1.34 & 5.38 & 0.2491 & 0.1506 & $0.512266 \pm 04$ & -5.49 \\
\hline 10679 & \multirow{2}{*}{ N. Mata } & 260 & 105 & 39 & 0.7399 & 209 & 7.30 & 37.10 & 1968 & 39 & 0.51 & -5.34 \\
\hline 106804 & & 263 & 94 & 8.129 & $0.747152 \pm 04$ & 0.712447 & 5.18 & 27.60 & 0.1877 & 0.1135 & $0.512191 \pm 03$ & -5.53 \\
\hline 106805 & \multirow{3}{*}{ Peraleda } & 255 & 126 & 5.873 & $0.735012 \pm 05$ & 0.709939 & 5.62 & 27.90 & 0.2014 & 0.1218 & $0.512221 \pm 03$ & -5.27 \\
\hline 106810 & & 277 & 170 & 4.726 & $0.729482 \pm 05$ & 0.709306 & 7.15 & 39.10 & 0.1829 & 0.1105 & 0.51220 & -5.15 \\
\hline 106811 & & 270 & 177 & 4.424 & $0.727392 \pm 06$ & 0.708507 & 7.68 & 44.90 & 0.1710 & 0.1034 & $0.512201 \pm 03$ & -4.95 \\
\hline 106815 & \multirow{2}{*}{ P. Arzobispo } & 414 & 63 & 19.183 & $0.795180 \pm 06$ & 0.713288 & 5.13 & 23.00 & 0.2230 & 0.1348 & $0.512212 \pm 03$ & -5.94 \\
\hline 106816 & & 393 & 49 & 23.437 & $0.806042 \pm 05$ & 0.705988 & 5.41 & 23.60 & 0.2292 & 0.1386 & $0.512229 \pm 03$ & -5.75 \\
\hline
\end{tabular}

Uncertainties for the ${ }^{87} \mathrm{Sr}^{186} \mathrm{Sr}$ and ${ }^{143} \mathrm{Nd} /{ }^{144} \mathrm{Nd}$ ratios are 2 sigma errors in the last two digits.

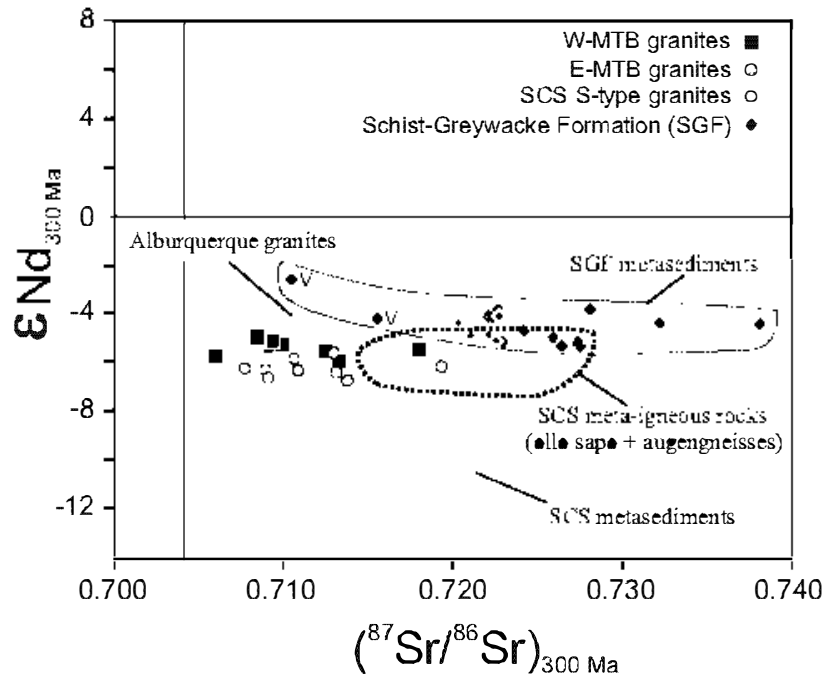

Fig. 10 Sr-Nd isotopic ratios at $300 \mathrm{Ma}$ for the W-MTB granites and related rocks. Fields for S-type granites from the Mora-Ventas Batholith (E-MTB) and the SCS are taken from Andonaegui (1990) and Villaseca et al. (1998b). Compositional field of the SGF is from Beetsma (1995), Ugidos et al. (1997) (shales), and Rodríguez-Alonso et al. (2004) (metavolcaniclastic rocks) (V). Isotopic field for the SCS meta-igneous rocks comprises: "ollo de sapo" (meta-volcanic) samples (Castro et al. 1999) and granite-derived orthogneisses (Villaseca et al. 1998a). Isotopic field for Alburquerque granites is taken from Menéndez and Bea (2004).

which have even higher initial $\varepsilon_{1: 1}$ values $(-3.4$ to -5.2 : Menéndez and Bea 2004).

Metasediments from the Veoproterozoic SchistGreywacke Formation (SGF) have significantly highor $\varepsilon_{1 *: 1}$ values when compared to other metasedimentary sequences in the Central Iberian Zone (Beetsma 1995; Rodrigue $\%$-Alonso et al. 2004). For example, metasediments from the SCS have $\varepsilon$, values mainly from -9.1 to -12.5 at $300 \mathrm{Ma}$ (Villaseca et al. 1998a) whereas those from the SGF have $\varepsilon_{1: 1}$ mainly in the range of -3.9 to -5.2

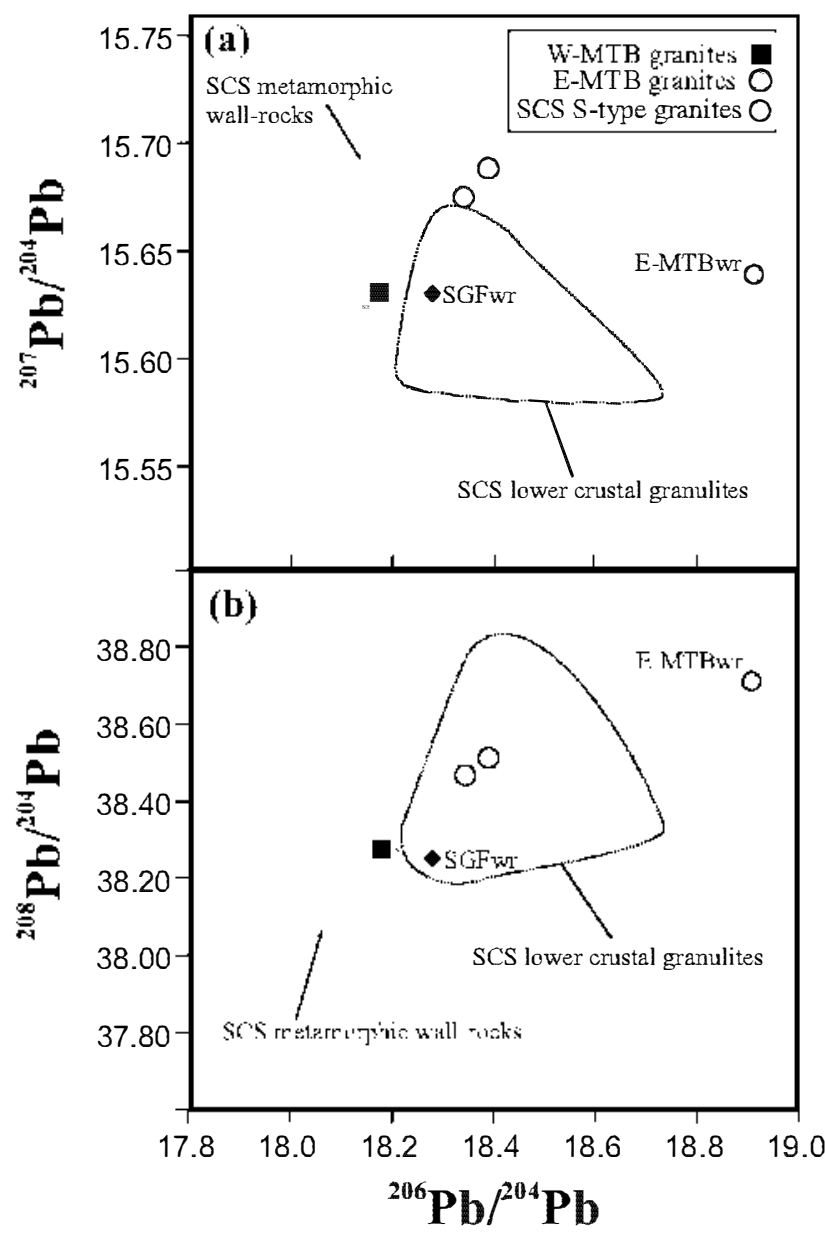

Fig. 11 Lead isotopic ratios of K-feldspars from S-type granites of central Spain: $\mathbf{a}-{ }^{206} \mathrm{~Pb} /{ }^{204} \mathrm{~Pb} v s^{207} \mathrm{~Pb} /{ }^{204} \mathrm{~Pb} . \mathbf{b}-{ }^{20}{ }^{20} \mathrm{~Pb} /{ }^{204} \mathrm{~Pb} v s^{208} \mathrm{~Pb} /{ }^{204} \mathrm{~Pb}$. Also shown is the field for the SCS lower crustal granulites (Villaseca et al. 2007) and the outcropping SCS metamorphic rocks (Villaseca et al. unpublished data). Age-corrected radiogenic $\mathrm{Pb}$ ratios for wholerock analyses are also included ( $S G F w r$ and $E-M T B w r)$. See text for further explanation. 
Tab. $6 \mathrm{~Pb}$ isotope data for S-type granites from central Spain

\begin{tabular}{|c|c|c|c|c|c|c|c|c|}
\hline Sample & Pluton & Material & $\mathbf{U}$ & Th & $\mathrm{Pb}$ & ${ }^{206} \mathrm{~Pb} /{ }^{204} \mathrm{~Pb}$ & ${ }^{207} \mathrm{~Pb} /{ }^{204} \mathrm{~Pb}$ & ${ }^{208} \mathrm{~Pb} /{ }^{204} \mathrm{~Pb}$ \\
\hline 106804 & N. Mata (W-MTB) & Kfsp & & & & 18.180 & 15.630 & 38.269 \\
\hline 80910 & Mora (E-MTB) & $\begin{array}{c}\text { whole } \\
\text { rock }\end{array}$ & 2.92 & 10 & 24 & $18.857^{*}$ & $15.637^{*}$ & $38.715^{*}$ \\
\hline 95916 & Alpedrete (SCS) & Kfsp & & & & 18.393 & 15.688 & 38.513 \\
\hline Y-82 & Hoyo Manz. (SCS) & Kfsp & & & & 18.345 & 15.674 & 38.465 \\
\hline
\end{tabular}

* Whole-rock data calculated at $300 \mathrm{Ma}$. Trace element contents in ppm.

(Beetsma 1995; Ugidos et al. 1997), which is much closer to reported values for western MTB granites (Fig. 10).

The only $\mathrm{Pb}$ isotopic analysis of a granite from the western MTB segment obtained in course of the present study (Tab. 6) shows the lowest isotopic ratios when compared to other peraluminous S-type granites from adjacent areas (Fig. 11). These low $\mathrm{Pb}$ isotopic ratios overlap with the field of outcropping metamorphic rocks from the CIZ, and are in contrast to the peraluminous granites from the SCS or from eastern MTB, which display radiogenic values more alike meta-igneous granulites from lower crustal levels to which they have been genetically related (Villaseca et al. 1999, 2007).

\section{Discussion}

\subsection{Conditions for magmatic andalusite-silli- manite crystallization}

The coexistence of andalusite and sillimanite in igneous felsic rocks is relatively uncommon and their origin remains controversial (e.g. Clarke et al. 2005). In some cases sillimanite is older than andalusite and the latter forms as crystallization proceeds during cooling (e.g. Macusani rhyolites, Pichavant et al. 1988) or as a consequence of nucleation around residual (xenolithic) sillimanite (D'Amico et al. 1981). However, as described for the studied MTB granites, it is more common to find the formation of sillimanite from andalusite. This polymorphic transformation has been interpreted in a number of ways: i) increasing temperature during progressive partial melting (e.g. Himalayan granites, Visonà and Lombardo 2002), ii) thermal metamorphism induced by younger granitic intrusions (e.g. Barrera et al. 1985), or iii) postmagmatic hydrothermal fibrolitic sillimanite transformation (e.g. Hassan Mohamud et al. 2002). Neither of these interpretations can be applied to the MTB granites. They are isolated plutonic massifs in which andalusite-sillimanite transformation appears in many samples within the pluton and it is unrelated to secondary processes or thermally metamorphosed areas. A late magmatic origin for both aluminium silicates is suggested by their euhedral appearance, chemical zoning and wide regional distribution, although unequivocal textural and chemical criteria to determine their origin are not straightforward (e.g. Clarke et al. 2005). However, these authors stated that chemical equilibrium with other granite minerals (e.g. biotite, muscovite) should reinforce the magmatic origin of aluminium silicates. In this respect, the correlation of $\mathrm{TiO}_{2}$ and $\mathrm{FeO}+\mathrm{MgO}$ contents with similar slopes of the tie lines between coexisting mica pairs suggests attainment of chemical equilibrium. The magmatic origin of muscovite is also consistent with the high $\mathrm{Na} /(\mathrm{Na}+\mathrm{K})$ ratios and projection in the $\mathrm{Mg}$ - TiNa diagram (Miller et al. 1981), shown by most of the analyzed muscovites (Fig. 4b), including the crystals surrounding andalusite.

Minor chemical impurities in andalusite structure enlarge its stability field towards higher temperatures (Kerrich and Speer 1988). Incorporation of $\mathrm{Fe}, \mathrm{Ti}, \mathrm{Mg}$ and $\mathrm{Mn}$ is higher in andalusite than in sillimanite which suggests a divariant rather than univariant equilibrium between both polymorphs (see also Kerrich 1990). Calculated $K d$

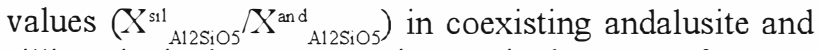
sillimanite in the MTB granites are in the range of 1.00 to 1.02 , which points to a shift of their equilibrium boundary

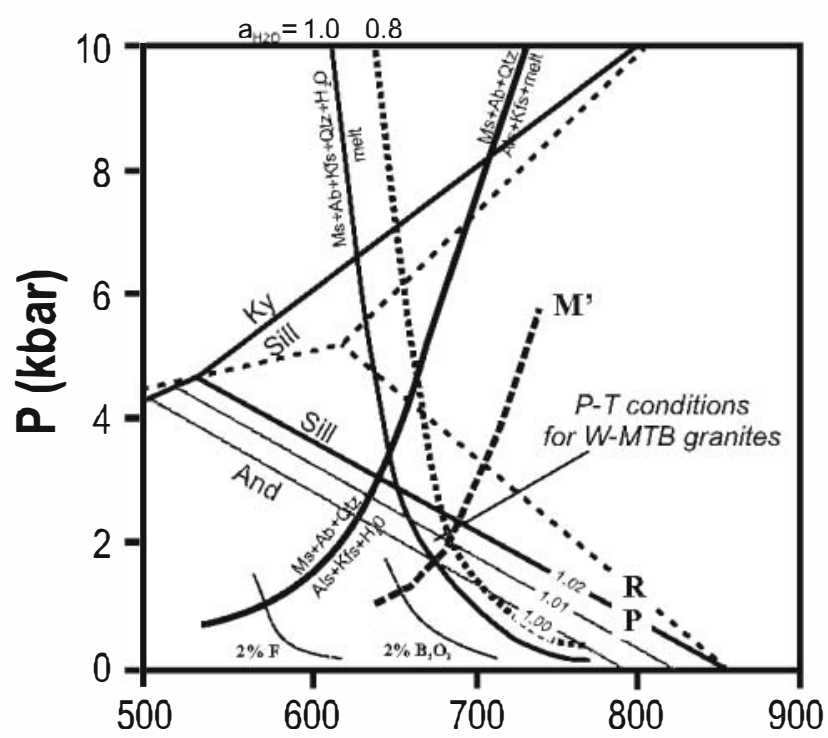

Fig. 12 A $P-T$ diagram showing the estimated crystallization conditions for the studied W-MTB granites. Isopleths of $\mathrm{K}_{\mathrm{eq}}\left(=\mathrm{X}_{\mathrm{A}}^{\mathrm{sil} 2 \mathrm{SiOs}} / \mathrm{X}_{\text {A12SiOs }}^{\text {and }}\right)$ for the andalusite-sillimanite equilibrium after Kerrick and Speer (1988). Andalusite-sillimanite-kyanite equilibria after (P) Pattison (1992) and (R) Richardson et al. (1969). Melting curves and reaction involving muscovite are taken from Johannes and Holtz (1996). M' muscovite reaction from D'Amico et al. (1981). Solidus curves for water-saturated haplogranitic systems with $\mathrm{F}$ and $\mathrm{B}$ are after Manning and Pichavant (1983). 
by $50^{\circ} \mathrm{C}$ upward (Kerrich 1990 drew this isopleth close to the Pattison 1992 equilibria) (Fig. 12). Moreover, the frequently observed andalusite chemical zoning towards rims poorer in minor elements indicates lowering of $K d$ values as crystallization proceeds (see also FernándezCatuxo et al. 1995). The incorporation of $\mathrm{Fe}, \mathrm{Ti}$ and $\mathrm{Mg}$ in andalusite and their progressive decrease in the felsic granitic magma, close to solidus conditions, makes andalusite unstable and sillimanite precipitates instead, or in combination with magmatic muscovite, depending on the water activity during crystallization. Thus, the transition from andalusite to sillimanite could be explained by changing local chemical conditions in residual granite melts rather than involving significant changes or reversals in magmatic $P-T$ conditions.

The possible equilibrium between andalusite-sillimanite and muscovite is rather restricted in a $P-T$ space (Fig. 12). This was discussed by D'Amico et al. (1981) who suggested that a deviation from the muscovite ideal formula (due to Ti-F-Na substitutions) would enlarge its stability field towards slightly lower $P$ conditions ( $M$ ' curve in Fig. 12). The restricted $P-T$ locus for late-magmatic crystallization of the proposed mineral assemblage in the western MTB granites remains around 2-3 kbar and $650-700{ }^{\circ} \mathrm{C}$.

\subsection{Granite sources}

The high peraluminosity of the western MTB granites and their remarkably low $\mathrm{CaO}$ contents when compared to the closely related S-type granites from the eastern MTB or the SCS suggest a major contribution from a metapelitic source (Chappell et al. 1991). This is in agreement with the suggestion of Sylvester (1998) who stated that $\mathrm{S}$-type peraluminous melts derived from pelites have lower $\mathrm{CaO} / \mathrm{Na}_{2} \mathrm{O}$ ratios $(<0.3)$ than melts produced from clay-poor (greywackeous) sources. The western MTB granites show $\mathrm{CaO} / \mathrm{Na}_{2} \mathrm{O}$ ratios between 0.19 and 0.52 (except for the Belvís leucogranites which are fractionated magmas), and this suggests mixed pelitic-greywackeous sources.

The high phosphorous content of the studied granites could be a consequence of their high peraluminosity as apatite dissolves more in such melts (Bea et al. 1992; Pichavant et al., 1992; Wolf and London 1994) or, alternatively, it can reflect inheritance from a P-rich source. Crustal sources with the highest phosphorous enrichments are pelitic shales (e.g. Gromet et al. 1984). The initially high phosphorous contents of metapelitic protoliths are reflected in the granitic melts that they produce, because phosphorous is more soluble in peraluminous compositions. The early crystallization of plagioclase, which removes calcium that would consume phosphorous in the form of apatite, contributes to a perphosphorous granite trend (London 2008).

Most of the P-rich granites of the CIZ are confined to the region where the Schist-Greywacke Formation occurs (Albalá, Alburquerque, Cabeza de Araya, Gata, Jálama, Trujillo, the western MTB) (Fig. 1). At the same time, the SGF is the CIZ metasedimentary formation richest in dispersed phosphate nodules and shows elevated $\mathrm{P}$ levels (IGME, 1989; Rodríguez-Alonso et al. 2004). Other isolated P-rich plutons in the CIZ include the Pedrobernardo Pluton from the SCS described as a classical perphosphorous granite (Bea et al. 1994) and similar bodies were studied in central Portugal (Neiva 1998). In the Tormes Dome area a large P-rich leucogranite batholith also occurs (López Plaza and López Moro 2003) but the more fractionated leucogranites show neither a marked perphosphorous trend nor any associated P-rich pegmatite fields as in the southern areas of the CIZ (e.g. P-rich granite pegmatites emplaced in the Pedroso de Acim and El Trasquilón granite cupolas, Gallego 1992; and also in the Alburqerque and Belvís de Monroy plutons: London et al. 1999; IGME 1983, respectively) (see Fig. 1 for locations). According to the current data, the strongly peraluminous granites of the southern half of the CIZ form the main P-rich pluton concentration in the whole Iberian Variscan Belt.

Isotope geochemistry of western MTB granites is consistent with a metasedimentary derivation from Neoproterozoic SGF rocks. The initial Nd ratios of the western MTB granites are similar to the isotopic ratios shown by mixed pelitic, greywackeous and metavolcaniclastic sources from the SGF (Fig. 10). The scarce Pb isotope data for the SGF metasediments (Beetsma 1995), and their age-corrected $\mathrm{Pb}$ isotope ratios (to $300 \mathrm{Ma}:{ }^{206} \mathrm{~Pb}$ / $\left.{ }^{204} \mathrm{~Pb}=18.28,{ }^{207} \mathrm{~Pb} /{ }^{204} \mathrm{~Pb}=15.63,{ }^{208} \mathrm{~Pb} /{ }^{204} \mathrm{~Pb}=38.26\right)$ are close to those obtained for the western MTB granite (Fig. 11). Isotopic data for Lower Palaeozoic felsic meta-igneous formations in the SCS (metavolcanics and metagranitic augen gneisses: Villaseca et al. 1998a; Castro et al. 1999 and references therein) have been also included in Fig. 10 for comparison, but their compositional field does not overlap with most of the studied W-MTB granites. Moreover, the absence of these meta-igneous formations in the southern part of the Central Iberian Zone makes the presence of meta-igneous rocks at depth difficult to imagine, especially when considering the eastern vergency of the whole SG metamorphic area (Fig. 1).

In Fig. 7 the compositional data of experimental melts derived from pelite-greywacke mixtures of the SGF (Fernández et al. 2008) are shown. Most of the biotite granites (Peraleda, N. Mata and Aldeanueva samples) have composition close to melts in low temperature experiments $\left(1000-1100^{\circ} \mathrm{C}\right)$ or produced at low melt 
fractions. By contrast, the more mafic granodiorites from Aldeanueva Pluton plot near the high-temperature experimental melts $\left(1200{ }^{\circ} \mathrm{C}\right)$ (Fig. 7). As such extremely high- $\mathrm{T}$ conditions are difficult to attain in continental collision areas, an alternative is that granodiorites, rich in surmicaceous and restitic enclaves, can contain a significant fraction of residual metasedimentary material. In any case, geochemical signatures of the studied W-MTB granites agree with their derivation by melting of SGF sources at crustal depths.

Most of the previous works on these P-rich plutons also support granite derivation from pelitic or peliticgreywackeous sources (Ramírez and Menéndez 1999: Menéndez and Bea 2014). Nevertheless. more recent studies suggest that mafic mantle-derived materials participated in the genesis of some of these plutons (e.g. the Cabeza de Araya Pluton - Castro and Corretge 2002 , García-Moreno et al. 2007), because the most mafic granite facies are less aluminous in composition and enclose hybrid mafic microgranular enclaves. In the studied WMTB granites the more mafic granites are, however, more peraluminous, plotting in the $h P$ fields of Villaseca et al. (1998b) (Fig. 7), thus supporting that metasedimentary sources were mainly involved in their origin.

\subsection{A segmented peraluminous granite batholith}

The MTB is a large peraluminous batholith which shows two contrasting S-type granites. The western segment includes the most peraluminous plutons with markedly higher $\mathrm{P}$ contents giving rise to strongly fractionated perphosphorous granites. The eastern segment of the MTB is built by peraluminous granites very close in composition to those of the SCS (Villaseca et al. 1998a) (Figs 7, 8 and 10).

The major differences in isotopic composition between the granites of the MTB are related to $\mathrm{Nd}$ and $\mathrm{Pb}$ ratios (Figs 10 and 11 ). The slightly higher initial $\mathrm{Nd}$ and the lower ${ }^{206} \mathrm{~Pb} /{ }^{204} \mathrm{~Pb}$ ratios in the western MTB granites suggest mainly metasedimentary sources, probably similar to the SGF, which presumably contains a grcaler proportion of juvenile magmatic components (Ugidos et al. 1997; Rodriguez-Alonso et al. 2004). On the contrary, the eastern MTB granites are closer in isotopic composition to meta-igncous granulites of lower crustal derivation, as stated in previous works (Villaseca et al. 1998a, 1999). However, this discussion should be considered as preliminary because the current isolopic $\mathrm{Pb}$ data are very scarce and the SGF and E-MTB (MoraVentas granite) samples were analyzed as whole-rock aliquots. which yield less accurate age-corrceted ratios. Further analytical work is needed to constrain the iso- topic composition of the MTB granites and associated metamorphic country rocks.

We propose that distinct crustal sources may have been involved in the origin of the peraluminous MTB and this implies that the S-type assignment is weakened. The combined use of chemical diagrams showing different peraluminosity and perphosphorous degrees could contribute to discrimination of different parental materials in the genesis of peraluminous granites (Fig. 13). In the A-B diagram the western MTB granites spread from $h P$ towards fP fields. On the other hand, the eastern MTB granites plot mostly in the $m P$ and $f P$ fields, but close to the proposed I-S boundary line of Villaseca et al. (1998b) (Fig. 7). The more mafic granites of the western MTB show a higher peraluminosity indicating more pelitic metasedimentary contribution, or alternatively, a higher melt fraction. In contrast, peraluminous granites with clear negative trends in the A-B plot suggest meta-igneous derivation or mixed crustal-mantle sources (Villaseca et al. 1998b).

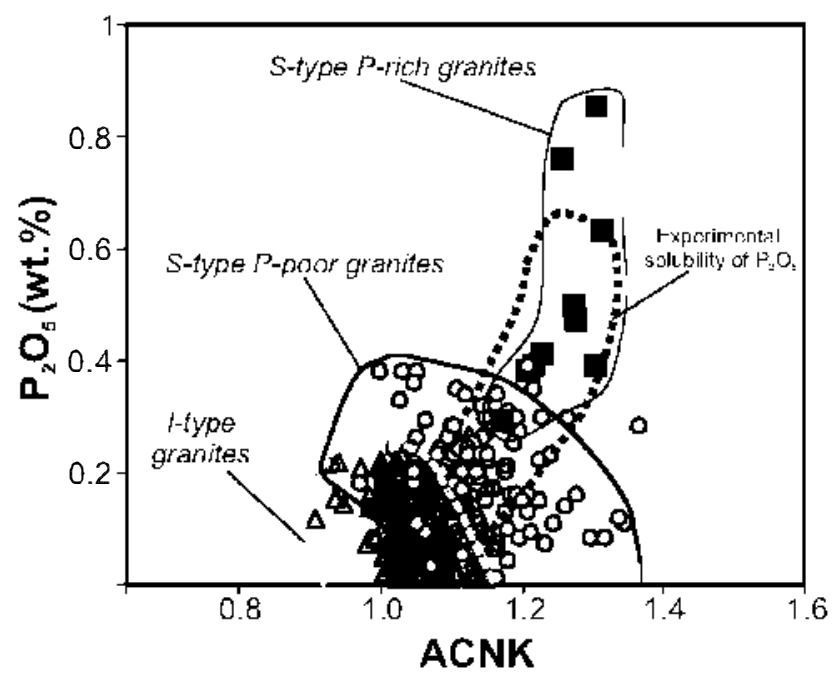

Fig. $13-\mathrm{A} / \mathrm{CNK}$ (molar $\mathrm{Al}_{2} \mathrm{O}_{3} \mathrm{i}\left(\mathrm{CaO}+\mathrm{Na}_{2} \mathrm{O}+\mathrm{K}_{2} \mathrm{O}\right)$ ) $\mathrm{P}_{2} \mathrm{O}$ (in wt. \%) of the CIZ granitic rocks. The three discriminant fields are: i) I-type granites from the SCS (Villaseca et al. 1998a), ii) S-type granites from the SCS and E-MTB (Andonaegui 1990; Villaseca et al. 1998a), iii) Stype P-rich granites from W-MTB. Field of experimental solubility of $\mathrm{P}_{2} \mathrm{O}_{\text {: }}$ in low-Ca peraluminous melts after Wolf and London (1994).

The MTB data demonstrate that there are different series of S-type granites. In this respect, the similarity in $\mathrm{P}$ enrichment of these peraluminous granites with the experimental solubility of $\mathrm{P}, \mathrm{O}$, as a function of the alumina saturation index of the melt (Fig. 13) supports the distinction of perphosphorous S-type granite series as the one that has other sinks for phosphate in the fractionation sequence (e.g. feldspars, Wolf and London 1994). 
The western MTB granites intruded into the SGF region whereas eastern plutons are more allochthonous, and penetrated the Lower Palaeozoic series. The Mora-Ventas Batholith (E-MTB) intruded along the Toledo listric fault (Fig. 1), a late Variscan E-W shear band which gave rise to a core complex-like structure, separating a large anatectic complex to the north (the ACT, Barbero and Villaseca 1992) from epizonal metamorphic series to the south. The ACT metasedimentary series displays the same geochemical signature as the SCS Neoproterozoic series (Villaseca et al. 1998a). Furthermore, the late-Variscan Toledo shear band is coincident with the general E-W granitic array of the MTB, suggesting tectonic control of granite generation and emplacement within the Montes de Toledo region. Shallower metasedimentary sources played a role in the genesis of granites of the western MTB, whereas deeper meta-igneous lithotypes (lower crustal felsic granulites) could have been involved in the granite genesis of its eastern segment.. Still, an evidence for a minor contribution of some mantle-derived mafic magmas can be postulated (presence of mafic bodies smaller than $20 \mathrm{~m}$ in length, and conspicuous mafic microgranular enclaves within the Mora-Ventas granites; Andonaegui 1990). The change from a ductile to brittle $\mathrm{E}-\mathrm{W}$ Caulting. from east to west across the MTB is perceptible, but the tectonic control and the main mechanism triggering partial melting and giving rise to this segmented batholith remains to be evaluated.

Acknowledgements. We acknowledge Alfredo Fernández Larios and José González del Tánago for their assistance with the electron microprobe analyses in the CAI of Microscopia Electrónica (UCM). Also José Manuel Fuenlabrada Pérez and José Antonio Hernández Jiménez from the CAI of Geocronología y Geoquímica (UCM) for their help in analysing samples by TIMS. We appreciate critical comments and suggestions by Antonio Castro, Migucl I.śpcz Plaza and David Dolejš, which greatly improved the quality of the manuscript. This work is included in the objectives of, and supported by, the CGL2004-02515/BTE and CGL200805952-CO2-01/BTE projects of the Ministerio de Educación y Ciencia of Spain, and the CCG07-UCM project.

\section{References}

Ábalos B, Carreras J, Druguet E, Escuder J, Gómez-Pugnaire MT, Lorenzo Álvarez S, Quesada C, RodríguezFERnÁndEZ R, GIL-IBARGUCHI JI (20012) Variscan and Pre-Variscan tectonics. In: GibBons W, MoReno T (eds) The Geology of Spain. Geol Soc London, pp 155-183

Abdel-Rahman A-F M (1994) Nature of biotites from alkaline, calc-alkaline and peraluminous magmas. J Petrol 35: 525-541
AndonaEgui P (1990) Geochemistry and Geochronology of the Granitoids South of Toledo. Unpublished PhD. Thesis, Complutense University, Madrid, pp 1-357 (in Spanish)

AndonAegui P, BARRERA.JL (1984)Petrology of two peraluminous granite series from Valdeverdeja-Aldeanueva de Barbarroya (Toledo). Bol Geol Min España 95: 165-183 (in Spanish)

Andonaegui P, Villaseca C (1998) Granites from the Mora-Gálvez Pluton (Toledo): an example of evolution by crystal fractionation. Bol R Soc Española Hist Nat 94: 17-31 (in Spanish)

Barbero L, Villaseca C (1992) The Layos granite, Hercynian complex of Toledo (Spain): an example of parautochthonous restite-rich granite in a granulitic area. Trans Roy Soc Edinb, Earth Sci 83: 127-138

Barrera JL, Bellido F, Klein E (1985) Contact metamorphism in synkinematic two-mica granites produced by younger granitic intrusions. Geol Mijnbouw 64 : 413-422

Bea F, Fershtater G, Corretgé LG (1992) The geochemistry of phosphorus in granite rocks and the effect of aluminium. Lithos 29: 43-56

Bea F, Pereira MD, Corretgé LG, Fershtater GB (1994) Differentiation of strongly peralum inous. perphosphorous granites: the Pedrobernardo Pluton, central Spain. Geochim Cosmochim Acta 58: 2609-2627

Bea F, Montero P, Molina JF (1999) Mafic precursors, peraluminous granitoids, and late lamprophyres in the Avila batholith: a model for the generation of Variscan batholiths in Iberia. J Geol 107: 399-419

Bea F, Montero P, Zinger T (2003) The nature, origin, and thermal influence of the granite source layer of Central Iberia. J Geol 111: 579-595

Betrisia JJ (1995) The late Proterozoic/Paleozoic and Hercynian crustal evolution of the Iberian Massif, $\mathrm{N}$ Portugal, as traced by geochemistry and $\mathrm{Sr}-\mathrm{Nd}-\mathrm{Pb}$ isolope systematics of pre-Hercynian terrigenous sediments and Hercynian granitoids. Unpublished Phl).Thesis, Virije Tniversitcit. Amsterdam, the Netherlands, pp 1-223

Capdevila R, Corretgé LG, Floor P (1973) Les granitoides Varisques de la Meseta lbérique. Bull Soc Géol France 15: 209-228

Castro A, Paitño-Douce A, Corretgé LG, De la Rosa J, El-Biad M, El-Hmidi H (1999) Origin of peraluminous granites and granodiorites, Iberian Massif, Spain: an experimental test of granite petrogenesis. Contrib Mineral Petrol 135: 255-276

Castro A, Corretgé LG (2010) Variscan granites. In: GibBONS W, MoReno T (eds) The Geology of Spain. Geol Soc London, pp 129-136

Chappell BW, White AJR, Wyborn D (1987) The importance of residual material(restite) in granite petrogenesis. J Petrol 28: 1111-1138 
Chappell BW, White AJR, Williams IS (1991)A transverse section through granites of the Lachlan Fold Belt. Second Hutton Symposium Excursion Guide. ABMR Record 1991/22, Canberra, pp 1-125

Clarke DB, Dorais M, Barbarin B, Barker D, Cesare B, Clarke G, El Baghdadi M, Erdmann S, Förster H-J, Gaeta M, Gottesmann B, Jamieson RA, Kontak DJ, Koller F, Gomes CL, London D, Morgan VI GB, Neves LJPF, Pattison DRM, Pereira. AJSC, Pichavant M, Rapela CW, Renno AD, Richards S, Roberis M, Rotiura A, SaAvedra J, Sial AN, Toselli AJ, Ugidos JM, Uher P, Villaseca C, Visona D, Whitney DL, Williamson B, WoOdard HH (2005) Occurrence and origin of andalusite in peralum inous felsic igneous rocks. J Petrol 46: 441-472

Clemens JD, Wall VJ (1981) Origin and crystallization of some peraluminous (S-type) granitic magmas. Canad Mineral 10: 111-131

D’Amico C, Rotiura A, Bargossi GM, Nannetti MC (1981) Magmatic genesis of andalusite in peraluminous granites. Examples from Eisgarn type granites in Moldanubicum. Rend Soc Italiana Mineral Petrol 38: 15-25

Debon F, Le Fort P (1983) A chemical-mineralogical classification of common plutonic rocks associations. Trans Roy Soc Edinb, Earth Sci 73: 135-149

Dias G, Leterrier J (1994) The genesis of felsic-mafic plutonic associations: a $\mathrm{Sr}$ and $\mathrm{Nd}$ isotopic study of the Hercynian Braga Granitoids Massif (Northern Portugal). Lithos 32: 207-223

Dias G, Leterrier J, Mendes A, Simões PP, Bertrand JM (1998) U-Pb zircon and monazite geochronology of synto post-tectonic Hercynian granitoids from the Central Iberian Zone (Northern Portugal). Lithos 45: 349-369

Erdmann S, Clarke DB, MacDonald MA (2004) Origin of chemically zoned and unzoned cordierites from the South Mountain and Musquodoboit batholiths. Trans Roy Soc Edinb, Earth Sci 95: 99-110

Fernández-Catuxo J, Corretgé LG, Suárez O (1995) Influence of minor elements on the stability of andalusite in granitic rocks from the Iberian Massif. Bol Soc Española Mineral 18: 55-71 (in Spanish)

FernándeZ-SuÁRez J, Dunning GR, Jenner GA, GutiérRezAlonso G (2000) Variscan collisional magmatism and deformation in NW Iberia: constraints from U-Pbgeochronology of granitoids. J Geol Soc, London 157: 565-576

Fernández C, Becchio R, Castro A, Viramonte JM, Moreno-Ventas I, Corretgé LG (2008) Massive generation of atypical ferrosilicic magmas along the Gondwana active margin: implications for cold plumes and back-arc magma generation. Gondwana Res 14: 451-473

GALLEGo M (1992) Las mineralizaciones de litio asociadas a magmatismo ácido en Extremadura y su encuadre en la zona Centro-Ibérica. Unpublished PhD. Thesis, Complutense University, Madrid, pp 1-323 (in Spanish)
García-Moreno O, Corretgé LG, Castro C (2007) Processes of assimilation in the genesis of cordierite leucomonzogranites from the Iberian Massif: a short review. Canad Mineral 45: 71-85

GREEN TH (1976) Experimental generation of cordierite- or garnet-bearing liquids from a pelitic starting composition. Geology 4: 85-88

Gromet LP, Dymek RF, Haskin LA, Korotev RL (1984) The "North American shale composite": its compilation, major and trace element characteristics. Geochim Cosmochim Acta 48: 2469-2482

Hassan Mohamud A, Casquet C, Pérez del Villar L, Cozar J, PELlicer MJ (2002) High temperature hydrothermal fibrolite in "El Payo granite" Cadalso-Casillas de Flores granitic complex(Salamanca-Cáceres, Spain). Geogaceta 32: $23-26$

IGME (1985) Spanish Geological Map, Sheet ${ }^{\circ}$ 653, Valdeverdeja. Serv Publ Minist Industria, Madrid.

IGME (1987) Spanish Geological Map, Sheet nº 652, Jaraicejo. Serv Publ Minist Industria, Madrid.

IGME (1989) Spanish Geological Map, Sheet nº 654, El Puente del Arzobispo. Serv Publ ITGE, Madrid.

Johannes W, Hol.tz F (1996) Petrogenesis and Experimental Petrology of Granitic Rocks. Springer-Verlag, Berlin, pp 1-335

KeMP AIS, Hawkesworth CJ (2003) Granitic perspectives on the generation and secular variation of the continental crust. In: RuDNICK RL (ed) The Crust, vol. 3 Treatise of Geochemistry. Elsevier-Pergamon, Oxford, pp 349-410

KerRick DM (1990) The $\mathrm{Al}_{2} \mathrm{SiO}_{5}$ polymorphs. Mineralogical Society of America Reviews in Mineralogy 22, pp $1-406$

KerRick DM, SpeEr JA (1988) The role of minor element solid solution on the andalusite-sillimanite equilibrium in metapelites and peraluminous granitoids. Amer J Sci 288: 152-192

Liñán E, Gozalo R, Palacios T, Gámez-Vintaned JA, Ugidos JM, Mayoral E (2002) Cambrian. In: GibBons W, Moreno T (eds) The Geology of Spain. Geol Soc London, pp 17-29

London D (2008) Pegmatites. Canad Mineral Spec Publ 10 , pp $1-347$

London D, Wolf MB, Morgan GB-VI, Gallego M (1999) Experimental silicate-phosphate equilibria in peraluminous granitic magmas, with a case study of the Alburquerque batholith at Tres Arroyos, Badajoz, Spain. J Petrol 40: 215-240

López Plaza M, López Moro FJ (2003) The Tormes Dome. Eurogranites in western Castilla y León. Guide Book, pp 1-192

Manning DAL, Pichavant M (1983) The role of fluorine and boron in the generation of granitic melts. In: ATHERTON MP, GRIBBLE CD (eds) Migmatites, Melting and Metamorphism, Shiva, Nantwich, pp 94-109 
MenÉndez LG, Bea F (2004) The Nisa-Alburquerque Batholith. In: VERA JA (ed) Geology of Spain, IGME-SGE, Madrid, pp 120-122 (in Spanish)

Miller CF, Stoddar EF, Bradfish LJ, Dollase WA (1981) Composition of plutonic muscovite: genetic implications. Canad Mineral 19: 25-34

Moreno Ventas I, Rogers G, Castro A (1995) The role of hybridization in the genesis of Hercynian granitoids in the Gredos Massif, Spain: inferences from Sr-Nd isotopes. Contrib Mineral Petrol 120: 137-149

Nachit H, Razafimahefa N, Stussi JM, Carron JP (1985) Composition chimique des muscovites et feldspaths potassiques dans les leucogranite du massif de Millesvaches (Massif Central Français). Comptes Rendus Acad Sci Paris 301: 813-818

NeIVA AMR (1998) Geochemistry of highly peraluminous granites and their minerals between Douro and Tamega valleys, northern Portugal. Chem Erde 58: 161-184

PATTISON DRM (1992) Stability of andalusite and sillimanite and the $\mathrm{Al}_{2} \mathrm{SiO}_{5}$ triple point: constraints from the Ballachulish aureole, Scotland. Amer Miner 86: 1414-1422

Pichavant M, Kontak DJ, Herrera JV, Clark AH (1988) The Miocene-Pliocene Macusani Volcanics, SE Peru. I. Mineralogy and magmatic evolution of a two-mica aluminosilicate-bearing ignimbrite suite. Contrib Mineral Petrol 100: 300-324

Pichavant M, Montel JM, Richard LR (1992) Apatite solubility in peraluminous liquids: experimental data and extension of the Harrison-Watson model. Geochim Cosmochim Acta 56: 3855-3861

RamíREZ JA, MenÉNDEZ LG (1999) A geochemical study of two peraluminous granites from south-central Iberia: the Nisa-Alburquerque and Jalama batholiths. Canad Mineral 63: 85-104

Reyes J, Villaseca C, Barbero L, Quejido AJ, Santos Zalduegui JF (1997) Description of a Rb, Sr, Sm and $\mathrm{Nd}$ separation method for silicate rocks in isotopic studies. I Congr Iber Geoquim Abstract Vol, pp 46-55 (in Spanish)

Richardson SW, Gilbert MC, Bell PM (1969) Experimental determination of kyanite-andalusite and andalusite-sillimanite equilibria: the aluminium silicate triple point. Amer J Sci 267, 259-272

Rodríguez-Alonso MD, Peinado M, López-Plaza M, Franco P, Carnicero A, Gonzalo JC (2004) Neoproterozoic-Cambrian synsedimentary magmatism in the Central Iberian Zone (Spain): geology, petrology and geodynamic significance. Int J Earth Sci 93: 897-920

Rossi JN, Toselli AJ, Saavedra J, Sial AN, Pellitero E, Ferreira VP (2002) Common crustal sources for contrasting peraluminous facies in the early Paleozoic
Capillitas Batholith, NW Argentina. Gondwana Res 5: 325-337

RudNick RL, GaO S (2003) Composition of the continental crust. In: RuDNick RL (ed) The Crust, vol. 3 Treatise of Geochemistry. Elsevier-Pergamon, Oxford, pp 1-64

Sun SS, McDonough WF (1989) Chemical and isotopic systematics of oceanic basalts; implications for mantle composition and processes. In: SAUNDERS AD, NORRY MJ (ed) Magmatism in the Ocean Basins. Geol Soc London Spec Publ 42: 313-345

SYLVESTER PJ (1998) Post-collisional strongly peraluminous granites. Lithos 45: 29-44

Ugidos JM, Valladares MI, Recio C, Rogers G, Fallick AE, Stephens WE (1997) Provenance of Upper Precambrian-Lower Cambrian shales in the Central Iberian Zone, Spain: evidence from a chemical and isotopic study. Chem Geol 136: 55-70

Valladares M I, Barba P, Ugidos JM (2002) Precambrian. In: Gibbons W, Moreno T (eds) The Geology of Spain. Geol Soc London, pp 7-16

Villaseca C, Barbero L (1994) Chemical variability of Al-Ti-Fe-Mg minerals in peraluminous granitoid rocks from central Spain. Eur J Mineral 6: 691-710

Villaseca C, Herreros V (2000) A sustained felsic magmatic system: the Hercynian granitic batholith of the Spanish Central System. Trans Roy Soc Edinb, Earth Sci 91: 207-219

Villaseca C, Barbero L, Rogers G (1998a) Crustal origin of Hercynian peralum inous granitic batholiths of central Spain: petrological, geochemical and isotopic $(\mathrm{Sr}, \mathrm{Nd})$ constraints. Lithos 43: 55-79

Villaseca C, Barbero L, Herreros V (1998b)A re-examination of the typology of peraluminous granite types in intracontinental orogenic belts. Trans R Soc Edinb, Earth Sci 89: 113-119

Villaseca C, Downes H, Pin C, Barbero L (1999) Nature and composition of the lower continental crust in central Spain and the granulite-granite linkage: inferences from granulite xenoliths. J Petrol 40: 1465-1496

Villaseca C, Orejana D, Paterson BA, Billstrom K, Pérez-SoBA C (2007) Metaluminous pyroxene-bearing granulite xenoliths from the lower continental crust in central Spain: their role in the genesis of Hercynian I-type granites. Eur J Mineral 19: 463-477

VisonA D, LomBardo B (2002) Two-mica and tourmaline leucogranites from the Everest-Makalu region (Nepal-Tibet). Himalayan leucogranite genesis by isobaric heating? Lithos 62: 125-150

Wolf MB, London D (1994) Apatite dissolution into peraluminous haplogranitic melts: an experimental study of solubilities and mechanisms. Geochim Cosmochim Acta 58: 4127-4145 